\title{
Learnability of Heterogeneous Misspecification Equilibrium ${ }^{1}$
}

\author{
Ryuichi Nakagawa ${ }^{2}$ \\ Faculty of Economics \\ Kansai University
}

This version: June 18, 2010

\footnotetext{
${ }^{1}$ This paper was presented at the Canadian Economics Association 44th Annual Conference (Quebec City, Canada, May 28, 2010) and the Japanese Economic Association 2010 Semiannual Meeting (Chiba, Japan, June 6, 2010), and will be presented at the Western Economic Association International 85th Annual Conference (Portland, Oregon, USA, July 2, 2010) and the European Economic Association 25th Annual Congress (Glasgow, UK, August 23-26, 2010). The author is grateful to Shutao Cao, George Evans, Ichiro Muto, and Takayuki Tsuruga for their helpful comments and suggestions. The author is solely responsible for any remaining errors. For financial support, the author thanks KAKENHI (No.20730139) and Nomura Foundation for Academic Promotion.

${ }^{2}$ Address: Faculty of Economics, Kansai University, 3-3-35 Yamate Suita, Osaka 564-8680, Japan. Phone: +81-6-6368-0590. Fax: +81-6-6339-7704. E-mail: ryu-naka@kansai-u.ac.jp. URL: http://www2.ipcku.kansai-u.ac.jp/ ryu-naka/.
} 


\title{
Learnability of Heterogeneous Misspecification Equilibrium
}

\begin{abstract}
This paper investigates the learnability of the equilibrium under adaptive learning with heterogeneous and misspecified models. Agents have imperfect and mutually different information sets of economic variables, so they form heterogeneous expectations under learning rules that are differently underparameterized. Under heterogeneous learning, the economy converges to a type of restricted perceptions equilibrium, which here is called a Heterogeneous Misspecification Equilibrium (HME). The paper finds that the HME is more learnable than the equilibrium under homogeneous and correctly specified learning rules that corresponds to a rational expectations equilibrium (REE); in other words, the stability conditions of the HME are less stringent than those of the REE. In a basic NK model with a Taylor-type monetary policy rule, for example, the central bank is allowed to choose a wider range of values of policy parameters to ensure the learnability of the HME than that permitted to ensure the learnability of the REE, that is, the Taylor principle. In addition, if the correlation between demand and supply shocks is low, the stability conditions of the HME become further less stringent so that the central bank might not have to address the learnability of the equilibrium.
\end{abstract}

JEL classification: C62; D83; E52

Keywords: Adaptive learning; Heterogeneous expectations; Taylor principle 


\section{Introduction}

The rational expectation is based on the extreme assumption that we know everything about the structure of an economy. However, we rarely have enough information about an economy to form rational expectations, and hence we estimate econometric models to forecast the future of the economy. In this situation, a rational expectations equilibrium (REE) is asymptotically realizable only if the equilibrium is adaptively learnable in the sense that our forecasts converge to rational expectations through recursive updating of our beliefs on the structure of the economy. Hence, the learnability of the equilibrium, or, in other words, the stability of the equilibrium under adaptive learning, has been considered one of the necessary conditions that the equilibrium must satisfy (see Evans and Honkapohja 2001; Bullard and Mitra 2002).

In practice, it is also improbable that any economic variables are observable so that agents can specify a correct form of econometric model that has the same form as the structure of the economy. Previous literature on this kind of learning often assumes that agents can observe any economic variables in forming their forecasts by adaptive learning. Actually, some exogenous variables such as demand and supply shocks, for example, are measurable only if the structure of the economy is correctly identified. Thus, agents might be constrained to form underparameterized econometric models by using only observable economic variables.

Furthermore, it is possible that agents use different econometric models and form heterogeneous expectations because the observability of economic variables can be different across agents. To be sure, any economic variable would necessarily be observed by someone; as a result, the economy as a whole would respond to all economic variables. However, some variables (e.g., preference shocks in households' utility, idiosyncratic shocks on firms) might be observable 
only for specific agents as private information. Each agent might be restricted to use a form of econometric model that is not only underparameterized but also different and that includes variables that are observable only for that agent.

This paper investigates equilibrium under heterogeneous and misspecified learning using a multivariate linear expectations model. Each type of agent is assumed to use a single form of heterogeneously underparameterized learning rule, using an imperfect and different information set of economic variables. We clarify whether the heterogeneous and misspecified expectations affect the characteristics and learnability of the equilibrium by comparing them with those of the equilibrium realized under learning with homogeneous and correctly specified expectations. Next, using a basic New Keynesian model with a Taylor-type monetary policy rule as an example, we examine the effect of heterogeneous learning on the conditions on policy parameters of the central bank aimed at keeping the equilibrium learnable.

The paper provides three main results. First, we confirm that the equilibrium under the heterogeneous and misspecified learning rules deviates from a REE, which is learnable under a homogeneous and correctly specified learning rule, and converges to a type of restricted perceptions equilibrium (RPE), which we term here the Heterogeneous Misspecification Equilibrium $(\mathrm{HME})^{1}$. The deviation of the HME from the REE is generated by two factors: first, the heterogeneity in learning that keeps the expectations of individual agents inconsistent with macroeconomic expectations; and second, the misspecification in learning that makes parameter estimates biased through correlation between the variables that are included in a learning rule and the variables that are not included. Next, we find that the HME is more learnable than

\footnotetext{
${ }^{1}$ The HME is defined by differentiating it from the RPE, because the original RPE does not incorporate heterogeneity in learning. See Evans and Honkapohja (2001) and Branch (2004) for the analysis of the RPE. Adam (2005) and Branch and McGough (2009) consider the RPE in basic NK models.
} 
the REE; in other words, the stability conditions of the HME are less stringent than those of the REE. Finally, as a result, in the basic NK model, the central bank is allowed to choose a wider range of values of policy parameters to ensure the learnability of the HME than that permitted to ensure the learnability of the REE, that is, the Taylor principle. In addition, if the correlation of demand and supply shocks is low, the stability conditions of the HME become further less stringent so that the central bank might not have to address the learnability of the equilibrium.

The related literature examines equilibria under heterogeneous and misspecified learning rules $^{2}$. Several studies investigate the heterogeneous expectations equilibrium (HEE) in which two different types of agents are constrained to have different information sets, so that agents of one type use an underparameterized econometric model while the others use a correctly specified model. Branch and McGough $(2004,2009)$ indicate that specifications that are determinate under rational expectations may possess multiple equilibria in the case of heterogeneity of expectations. Guse (2005) shows that the learnability of the HEE is affected by the proportion of agents that use each different learning model. Berardi (2007) finds the conditions under which the HEE is learnable while the REE is not under a correctly specified learning rule. Berardi (2009b) argues that heterogeneity plays an important role in the central bank's policy

\footnotetext{
${ }^{2}$ See Giannitsarou (2003) and Honkapohja and Mitra (2006) to find the stream of research on heterogeneous learning. Heterogeneous learning has also been discussed from two other viewpoints. First, Evans and Honkapohja (1996, 2001), Giannitsarou (2003), and Honkapohja and Mitra (2006) investigate stability conditions in a situation in which agents have different initial beliefs about the parameters in their homogeneous econometric models. They find that the heterogeneity in adaptive learning gradually disappears, and the equilibrium is as learnable as to converge to a REE. That is, this heterogeneity has no persistent effect on the stability of equilibrium. Second, Evans et al. (2001) and Honkapohja and Mitra (2005) examine learnability in a situation in which agents have different learning algorithms (e.g., heterogeneity in updating functions or gain parameters). Their heterogeneity delays the updating of estimated parameters in their models, but heterogeneous updating rules become asymptotically homogeneous so that the equilibrium converges to the REE. These studies all investigate heterogeneous learning, but their findings mostly coincide in that the equilibrium under those types of heterogeneous learning converges to the REE.
} 
implementation using a basic New Keynesian model.

Other relevant literature considers the misspecification equilibrium (ME), in which agents choose between a list of different underparametrized econometric models and base their selections on relative forecast performance ${ }^{3}$ Branch and Evans (2006) imply that the learnability of the ME depends on the method for estimating the forecasting performance of the models. Branch and Evans (2007) show that multiple MEs may be learnable under least squares learning in a Lucas-type monetary model. On the other hand, Berardi (2009a) finds that sunspot equilibria that can emerge in the HEE specification are not learnable under the heterogeneity of agents choosing the best-performing models.

This paper contributes to related research in three main aspects. First, the paper introduces an alternative specification of the heterogeneous learning process, the HME specification, in which each type of agents are constrained to use a single form of different and underparameterized learning rule. This specification will make it possible to investigate adaptive learning in economies with private information or when different agents are concerned with different local variables $^{4}$. Second, this paper provides analytical results of the characteristics and learnability of the HME that emerge in a multivariate linear expectations model. Thus, our results are directly applicable to the analysis of the learnability of a variety of linear macroeconomic models under

\footnotetext{
${ }^{3}$ Brock and Hommes (1997) is the first study to investigate equilibria under the heterogeneity of agents choosing the best-performing models. Branch and Evans (2006) extend the model to the ME model where agents do not choose between a costly accurate forecast and a costless unsophisticated forecast, but choose between equally underparametrized costless models.

${ }^{4}$ This issue is suggested as a future work by Honkapohja and Mitra (2006). In contrast, the related specifications mentioned above may be interpreted to incorporate the assumption that agents exist that have a full information set of economic variables. In the HEE specification, some of the agents are assumed to have enough information about economic variables to form a correctly specified learning model. In the ME specification, agents are also assumed to have enough information to allow them to arbitrarily choose an optimal learning rule. Thus, our specification can be differentiated from related ones in that each type of agent is assumed to have private information.
} 
heterogeneously misspecified learning rules. Third, using a basic NK model, the paper shows the effect of the correlation of demand and supply shocks on the learnability of an equilibrium under heterogeneous learning, a topic that has been rarely discussed in the literature.

The paper is structured as follows. The next section introduces our model. Section 3 investigates the evolution of the economy under a homogeneous and correctly specified learning rule. Section 4 introduces the HME specification and examines changes in the evolution of the economy. Section 5 calibrates the effect of the heterogeneous learning using a basic NK model. Finally, we present our conclusions.

\section{Model}

In this section, we introduce a multivariate linear expectations model to obtain an equilibrium for agents to form rational (or irrational) expectations:

$$
\left\{\begin{array}{l}
y_{t}=B y_{t+1}^{e}+C w_{t} \\
w_{t}=\Phi_{n} w_{t-1}+v_{t}
\end{array}\right.
$$

$y_{t}$ is a $m \times 1$ vector of endogenous variables. $w_{t}$ is a $n \times 1$ vector of autoregressive exogenous variables. $v_{t}$ is a $n \times 1$ vector of shocks that may correlate with one another and drive the stochastic processes of the exogenous variables. $e$ is the operator of rational (or irrational) expectation. $B$ is a $m \times m$ coefficient matrix of endogenous variables, and $C$ is a $m \times n$ coefficient matrix of exogenous variables. $\Phi_{n}$ is a $n \times n$ matrix of autoregressive coefficients of exogenous variables. We add several assumptions to simplify later analysis as follows.

\section{Assumption 1}


1. $\Phi_{n}$ is a diagonal matrix, whose diagonal elements are in $(0,1)$ and observable.

2. $\operatorname{det}\left(I_{m}-B\right) \neq 0$.

Assumption 1.1 is a standard assumption in the analysis of adaptive learning. Assumption 1.2 avoids that the equilibrium $y_{t}$ could be indeterminate to exogenous variables $w_{t}$.

If agents form rational expectations conditional on all available information, there can be a uniquely stable REE as a fundamental equilibrium. The easiest methodology by which to obtain the reduced form solution is to apply the method of undetermined coefficients. We guess a minimal state variable (MSV) solution to be

$$
y_{t}=a+c w_{t}
$$

substitute it into the system (1), and obtain the solutions of $(a, c)$.

$$
\left\{\begin{array}{l}
\bar{a}=\mathbf{0} \\
\bar{c}=B \bar{c} \Phi_{n}+C .
\end{array}\right.
$$

If the REE (3) is learnable, agents' beliefs of the system (1) that are formed through adaptive learning are able to converge to the REE.

\section{Homogeneous and Correctly Specified Learning Rule}

Next, we consider the economy in which agents form homogeneous beliefs about the system (1) through adaptive learning. We assume that all agents do not have enough information about the system (1) to form rational expectations and that agents have only the data of $y_{t}$ and $w_{t}$ up

to time $t,\left\{y_{s}, w_{s}\right\}_{s=1}^{t}$. Using the available data, agents will specify an adaptive learning rule, 
that is, a perceived law of motion (PLM), by which to form their beliefs and expectations of the future economy.

\subsection{Dynamics}

If all agents have a information set of economic variables $\left\{y_{s}, w_{s}\right\}_{s=1}^{t}$, they can specify a correct form of PLM that is in the same form as the MSV solution (2),

$$
y_{t}=a+c w_{t}+\varepsilon_{t},
$$

where $a$ is a $m \times 1$ vector of constant terms, $c$ is a $m \times n$ coefficient matrix for $w_{t}$, and $\varepsilon_{t}$ is a $m \times 1$ vector of error terms that are believed to be white noises. We define a parameter matrix by $\phi^{\prime}=(a, c)^{5}$.

Under the PLM (4), agents' expectations $y_{t+1}^{e}$ are established by

$$
\begin{aligned}
y_{t+1}^{e} & =a+c w_{t+1}^{e} \\
& =a+c \Phi_{n} w_{t} .
\end{aligned}
$$

Substituting (5) into the system (1), an actual law of motion (ALM) of the economy is determined by

$$
y_{t}=B a+\left(B c \Phi_{n}+C\right) w_{t}
$$

In real-time learning, agents recursively update the estimates $\phi^{\prime}=(a, c)$ using newly available data every time. Evans and Honkapohja (2001, chapter 2) have introduced the E-stability

\footnotetext{
${ }^{5}$ We focus on the PLM of the MSV form that does not depend on sunspot terms. To the system (1), there could be nonexplosive sunspot solutions, which might be learnable under adaptive learning if agents specified a PLM including sunspot terms. Evans and McGough (2005) indicate that there exist sunspot solutions that are learnable under the PLMs with sunspot terms. However, for that purpose, they impose several strong conditions on the characteristics of the sunspot terms. Duffy and Xiao (2007) show that in a general real business cycle model, sunspot solutions are always unstable under adaptive learning. Berardi (2007) focuses on the PLMs without sunspot terms in a HEE model. Hence, we consider that sunspot solutions would be unstable under adaptive learning.
} 
principle that if agents conduct recursive least-squares learning, the local convergence of the estimates $\phi$ is governed by the following ordinary differential equation (ODE)

$$
\frac{d}{d \tau}(a, c)=T(a, c)-(a, c)
$$

where $T(a, c) \equiv\left(T_{a}(a), T_{c}(c)\right)=\left(B a, B c \Phi_{n}+C\right)$, which is equivalent to a mapping from the PLM (4) to the ALM (6). $\tau$ denotes notional time. As a result, the fixed point of the ODE (7),

$$
\left\{\begin{array}{l}
\bar{a}=\mathbf{0} \\
\bar{c}=B \bar{c} \Phi_{n}+C
\end{array}\right.
$$

can be the equilibrium under recursive least-squares learning.

If agents' beliefs $\phi^{\prime}=(a, c)$ converge to the fixed point $(8), \bar{\phi}=(\bar{a}, \bar{c})$ is a unique and stable equilibrium under the correctly specified learning rule (4). Furthermore, the equilibrium (8) is equivalent to the REE (3); the REE can be considered as a convergence limit of the equilibrium realized under the homogeneous and correctly specified learning rule.

\subsection{Stability Conditions}

For agents' beliefs to converge to the fixed point (8), the ODE (7) has to be locally stable in the neighborhood of the fixed point. As Evans and Honkapohja (2001, Proposition 5.6) have confirmed, the ODE (7) is locally stable if and only if its Jacobians,

$$
\begin{aligned}
D\left(T_{a}(a)-a\right) & =B-I_{m} \\
D\left(T_{c}(c)-c\right) & =\Phi_{n}^{\prime} \otimes B-I_{m n}
\end{aligned}
$$

have all negative real parts of eigenvalues, in other words, the largest values of the real parts of the eigenvalues of $B$ and $\Phi_{n}^{\prime} \otimes B$ are less than unity. Hereafter, to simplify expositions, $\lambda[X]$ 
denotes the largest value of the real parts of the eigenvalues of a matrix $X$. Then, the above conditions are expressed by $\lambda[B]<1$ and $\lambda\left[\Phi_{n}^{\prime} \otimes B\right]<1$.

To be precise, the stability of the ODE (7) solely depends upon whether $\lambda[B]<1$. Notice that the eigenvalues of $\Phi_{n}^{\prime} \otimes B$ in (10) consist of all combinations of the products of the eigenvalues of $\Phi_{n}$ and those of $B$. Furthermore, by Assumption 1.1, the eigenvalues of $\Phi_{n}$ are real and in $(0,1)$. Then, if matrix $B$ satisfies $\lambda[B]<1, B$ automatically satisfies $\lambda\left[\Phi_{n}^{\prime} \otimes B\right]<1$. Thus, the stability condition is solely expressed by $\lambda[B]<1$.

What if agents recognize that there are no constant terms in the system (1) from the beginning? Then, they specify a PLM that excludes constant terms, instead of (5).

$$
y_{t}=c w_{t}
$$

In this case, the ODE (7) is locally stable if and only if the Jacobian (10) has all negative real parts of eigenvalues, that is, $\lambda\left[\Phi_{n}^{\prime} \otimes B\right]<1$.

Therefore, the stability conditions of the REE are summarized as follows.

Proposition 1 In the system (1), if agents learn adaptively by specifying homogeneous and correctly specified models, a possible stable equilibrium is unique and equivalent to the REE (8). The equilibrium is locally stable if and only if:

1. under the PLM with constant terms (4), $\lambda[B]<1$;

2. under the PLM without constant terms (11), $\lambda\left[\Phi_{n}^{\prime} \otimes B\right]<1$. 
Further, as is shown above, the condition $\lambda\left[\Phi_{n}^{\prime} \otimes B\right]<1$ is less restrictive for $B$ than $\lambda[B]<1$ is. Then, the real parts of the eigenvalues of matrix $B$ have a wider range of values under the PLM without constant terms (11).

Corollary 1 If agents recognize that no constant terms exist in the system (1) from the beginning and specify a PLM without constant terms (11), stability conditions are less restrictive than otherwise.

The reason is intuitively understandable. The PLM (11) is more correctly specified than the PLM (4) because the system (1) has no constant terms. Then, if agents originally recognize the non-existence of constant terms in the system and use (11), the expectations of agents become more accurate. As a result, restrictions on the matrix $B$ to ensure the learnability of the equilibrium are relaxed ${ }^{6}$.

\section{Heterogeneous and Misspecified Learning Rules}

In the following, we consider that agents have heterogeneous beliefs on the structure of the economy by specifying different PLMs. The economy depends upon a number of economic variables. However, agents are rarely able to observe all of those variables, in particular, exogenous variables such as structural shocks. Then, agents might be constrained to respond to specific exogenous variables that are observable only for them.

\footnotetext{
${ }^{6}$ Bullard and Mitra (2002, Appendix B) also find that in a basic NK model with a Taylor-type nominal interest rate rule of the central bank, conditions on coefficients in the rule to ensure the learnability of the equilibrium become less restrictive if agents specify a PLM without constant terms.
} 
Suppose that there are $n$ types of agents. The population of each type is $\frac{1}{n}$. Agents of type $i \in\{1, \ldots, n\}$ are able to observe a single exogenous variable $w_{i, t}$ in $w_{t}=\left\{w_{i, t} \mid i=1, \ldots, n\right\}$. Then, even though agents form expectations under adaptive learning, they cannot specify a correct form of PLM that responds to all exogenous variables. In this setting, the number $n$ represents not only the quantity of exogenous variables, but also the heterogeneity in learning. If $n=1$, this heterogeneity disappears, and the system is reduced to the previous one via homogeneous learning.

We decompose the notations of vectors and matrices in (1) to notations in line with heterogeneous learning.

$$
w_{t}=\left(w_{1, t}, \ldots, w_{n, t}\right)^{\prime}, v_{t}=\left(v_{1, t}, \ldots, v_{n, t}\right)^{\prime}, C=\left(C_{1}, \ldots, C_{n}\right), \Phi_{n}=\left(\begin{array}{ccc}
\varphi_{1} & \cdots & 0 \\
\vdots & \ddots & \vdots \\
0 & \cdots & \varphi_{n}
\end{array}\right) .
$$

Note that $w_{i, t}, v_{i, t}$, and $\varphi_{i} \in(0,1)$ are all scalars. $C_{i}$ is a $m \times 1$ coefficient vector of $w_{i, t}$. We define the standard deviation of $w_{i}$ as $\sigma_{i i}(\neq 0)$ and the correlation matrix of $w_{t}$ as $\Gamma_{n} \equiv\left(\rho_{i j}\right)_{n \times n}$ $\left(\rho_{i i}=1, \rho_{i j}=\rho_{j i},\left|\rho_{i j}\right| \leq 1\right)$. In order to simplify our later analysis, we make the assumption of $\Gamma_{n}{ }^{7}$.

Assumption $2 \Gamma_{n}$ is a nonnegative matrix, in which $\rho_{i j} \geq 0$ for any $i, j \in\{1, \ldots, n\}$.

This assumption has no effect on our analysis.

Further, we introduce the notation of a $n \times n$ idempotent matrix $\Xi_{n} \equiv \frac{1}{n}\left(x_{i j}\right)\left(x_{i j}=1\right.$ for any $i, j)$. Note that eigenvalues of $\Xi_{n}$ are 0 and 1 .

\footnotetext{
${ }^{7}$ Even if a correlation between $w_{i}$ and $w_{j}, \rho_{i j}$, is negative, this becomes positive by changing the sign of $w_{i}$ to $-w_{i}$ and redefining the correlation between $-w_{i}$ and $w_{j}$ as $\rho_{i j}$. By iterating this transformation for all possible negative correlations, we can make all correlations nonnegative.
} 


\subsection{Dynamics}

As agents $i$ observe a different exogenous variable $w_{i, t}$, the agents form a heterogeneous belief by specifying a different and underparameterized PLM.

$$
y_{t}=a_{i}+c_{i} w_{i, t}+\varepsilon_{i, t}
$$

$a_{i}$ and $c_{i}$ are $m \times 1$ vectors of constant terms and coefficients. $\varepsilon_{i, t}$ is a $m \times 1$ vector of error terms that are believed to be white noises ${ }^{8}$. Then, the expectations of agents $i$ are made as

$$
y_{i, t+1}^{e}=a_{i}+c_{i} \varphi_{i} w_{i, t}
$$

Then, under the heterogeneous PLMs (12), the average PLM of the economy is given as follows ${ }^{9}$.

$$
\begin{aligned}
y_{t} & =\frac{1}{n} \sum_{i=1}^{n}\left(a_{i}+c_{i} w_{i, t}+\varepsilon_{i, t}\right) \\
& =\frac{1}{n}\left\{\left(I_{m}, \ldots, I_{m}\right)\left(\begin{array}{c}
a_{1} \\
\vdots \\
a_{n}
\end{array}\right)+\left(c_{1}, \ldots, c_{n}\right)\left(\begin{array}{c}
w_{1, t} \\
\vdots \\
w_{n, t}
\end{array}\right)+\left(I_{m}, \ldots, I_{m}\right)\left(\begin{array}{c}
\varepsilon_{1, t} \\
\vdots \\
\varepsilon_{n, t}
\end{array}\right)\right\} \\
& =\frac{1}{n}\left(I_{m}, \ldots, I_{m}\right) a+\frac{1}{n} c w_{t}+\frac{1}{n}\left(I_{m}, \ldots, I_{m}\right) \varepsilon_{t} .
\end{aligned}
$$

where $a \equiv\left(a_{1}^{\prime}, \ldots, a_{n}^{\prime}\right)^{\prime}, c \equiv\left(c_{1}, \ldots, c_{n}\right)$, and $\varepsilon_{t} \equiv\left(\varepsilon_{1, t}^{\prime}, \ldots, \varepsilon_{n, t}^{\prime}\right)^{\prime} 10$. Under heterogeneous expecta-

\footnotetext{
${ }^{8} \mathrm{As}$ a result, the equilibrium derived in this section is not a consistent expectations equilibrium, which is defined by Hommes and Sorger (1998), that requires that agents correctly perceive all autocorrelations of $\varepsilon_{t}$. Evans and Honkapohja (2001, chapter 13.1.3) argue that if we assume $w_{t}$ to be iid, the equilibrium under misspecified learning becomes the consistent expectations equilibrium.

${ }^{9}$ Following Branch and Evans (2006), who assume agents to specify heterogeneous and misspecified PLMs, we define the average PLM of the overall economy as the weighted average of PLMs of heterogeneous agents. We assume necessary conditions for the linear combination of heterogeneous expectations to hold in our model, given by Branch and McGough (2009). On the other hand, Berardi (2007) introduces structural heterogeneity, under which heterogeneous PLMs contribute to the average PLM of the economy to different degrees.

${ }^{10}$ Note that the definition of $a$ is changed from the definition under homogeneous learning, (4). However, to avoid complexity in our analysis, we use the same notation as before for the current constant term matrix $\left(a_{1}^{\prime}, \ldots, a_{n}^{\prime}\right)^{\prime}$.
} 
tions (13), the average expectation $y_{t+1}^{e}$ is obtained by

$$
\begin{aligned}
y_{t+1}^{e} & =\frac{1}{n} \sum_{i=1}^{n} y_{i, t+1}^{e} \\
& =\frac{1}{n}\left\{\left(I_{m}, \ldots, I_{m}\right) a+c \Phi_{n} w_{t}\right\} .
\end{aligned}
$$

Thus, under heterogeneous learning, individual expectations $y_{i, t+1}^{e}$ continue to be different from the average expectation $y_{t}^{e}$, except the economy is at the steady state where $y_{t+1}^{e}=y_{i, t+1}^{e}=\mathbf{0}$ for any $i$.

Substituting (15) into the system (1), an ALM of the economy is obtained as

$$
\begin{aligned}
y_{t} & =B y_{t+1}^{e}+C w_{t} \\
& =B\left(\frac{1}{n}\left\{\left(I_{m}, \ldots, I_{m}\right) a+c \Phi_{n} w_{t}\right\}\right)+C w_{t} \\
& =\frac{1}{n} B\left(I_{m}, \ldots, I_{m}\right) a+\left(\frac{1}{n} B c \Phi_{n}+C\right) w_{t} .
\end{aligned}
$$

The ALM (16) indicates that the economy as a whole responds to any exogenous variable that is observable only for a certain type of agents.

Next, we have to calculate the real-time learning processes of each type of agents. As mentioned in Section 3.1, in real-time learning, type $i$ agents recursively update the estimates $\phi_{i}^{\prime}=\left(a_{i}, c_{i}\right)$ using newly available data every time. However, under heterogeneous learning, the E-stability principle is not satisfied; that is, even if agents conduct recursive least-squares learning, the local convergence of $\phi_{i}$ is not governed by an ODE that could be made by mapping from the PLM (14) to the ALM (16).

If type $i$ agents update estimates $\phi_{i, t}^{\prime}=\left(a_{i, t}, c_{i, t}\right)$ in time $t$ by least-squares projection of $y_{t}$ on $z_{i, t}^{\prime} \equiv\left(1, w_{i, t}\right), \phi_{i, t}^{\prime}$ must satisfy an orthogonality condition,

$$
E z_{i, t}\left(y_{t}-\phi_{i, t}^{\prime} z_{i, t}\right)=\mathbf{0}
$$


Individual agents do not estimate fully accurately, but their estimations are as accurate as possible given the available information up to time $t,\left\{y_{s}, w_{s}\right\}_{s=1}^{t}$. The operator $E$ denotes the expectation of variables, for $\phi_{i, t}$ fixed, taken over the invariant distributions of the exogenous variables $w_{t}$.

After obtaining the estimates to satisfy (17) for all $i$, we can define the dynamics of the estimated parameters $\left\{\phi_{i}\right\}_{i=1}^{n}$, the derivation of which is described in Appendix A, as follows.

Definition 1 The economy is defined as the stationary stochastic process of $y_{t}$ following the system (1). Under heterogeneous and misspecified learning, the PLM of type $i$ agents is formed by (12). Then, the dynamics of the estimates of all types, $\left\{\phi_{i}\right\}_{i=1}^{n}$, is subject to the following ordinary differential equations.

$$
\left\{\begin{array}{l}
\frac{d a}{d \tau}=T_{a}(a)-a, \\
\frac{d c}{d \tau}=T_{c}(c)-c,
\end{array}\right.
$$

where

$$
\begin{aligned}
T_{a}(a) & \equiv\left(\Xi_{n} \otimes B\right) a \\
T_{c}(c) & \equiv\left(\frac{1}{n} B c \Phi_{n}+C\right) \Psi_{n}, \\
\Psi_{n} & \equiv \operatorname{diag}\left(\sigma_{i i}\right) \Gamma_{n} \operatorname{diag}\left(\sigma_{i i}\right)^{-1} .
\end{aligned}
$$

Under Assumption 1.2, the equilibrium under heterogeneous learning is a fixed point of the ODE (18).

$$
\begin{aligned}
\bar{a} & =\mathbf{0}, \\
\bar{c} & =\left(\frac{1}{n} B \bar{c} \Phi_{n}+C\right) \Psi_{n} .
\end{aligned}
$$


In this paper, the equilibrium $(19,20)$ is termed the Heterogeneous Misspecification Equilibrium (HME).

Compared with the REE (8), the HME deviates in terms of the fixed point of $\bar{c}$. We can find two reasons for the divergence. First, the deviation of $\bar{c}$ is generated by the heterogeneity in expectations, which appears as $\frac{1}{n}$ in (20). Agents never recognize that individual types of agents follow heterogeneous learning that keeps the expectations of each type inconsistent with the average expectation of the economy ${ }^{11}$. Second, the misspecification in forming PLMs also makes the HME deviate from the REE. This effect is reflected by $\Psi_{n}$, the $(i, j)$ element of which is $\sigma_{i i}^{-1} \rho_{i j} \sigma_{j j}$, the least-squares estimate of the coefficient in the equation where $w_{j}$ is regressed upon $w_{i}$. The estimates $c_{t}$ have omitted variable bias so that the values of those estimates automatically deviate from the values that are estimated under the correctly specified PLM $(4)^{12}$. If $\rho_{i j}=0$ for any $i \neq j$, then $\Psi_{n}=I_{n}$, and the bias would disappear. In reality, $\rho_{i j} \neq 0$ for some $i$ and $j$ so that, under the misspecified PLMs, explanatory variables and error terms correlate so as to make the estimates biased. Consequently, the dynamics of the HME deviates from the dynamics of the $\mathrm{REE}^{13}$.

\subsection{Stability Conditions}

Next, we consider conditions for the HME to be stable under heterogeneous learning.

\footnotetext{
${ }^{11}$ Under homogeneous learning $(n=1)$, individual agents form the same expectations. In this case, individual expectations are automatically consistent with the average expectation, even if each agent is unable to apprehend the formation of expectations by other agents.

${ }^{12}$ Omitted variable bias and its effect on the dynamics of the economy are discussed by Branch and Evans (2007).

${ }^{13}$ Note that even if exogenous variables were not correlated with one another $\left(\Psi_{n}=I_{n}\right)$, the dynamics of the HME continues to be divergent from that of the REE as long as the heterogeneity in learning exists $(n \neq 1)$.
} 
The ODE (18) is locally stable if and only if its Jacobians,

$$
\begin{aligned}
D\left(T_{a}(a)-a\right) & =\Xi_{n} \otimes B-I_{m n} \\
D\left(T_{c}(c)-c\right) & =\left(\Phi_{n}\left(\frac{1}{n} \Psi_{n}\right)\right)^{\prime} \otimes B-I_{m n}
\end{aligned}
$$

have all negative real parts of eigenvalues at the fixed point $(19,20)$, in other words, $\lambda\left[\Xi_{n} \otimes B\right]<$ 1 and $\lambda\left[\left(\Phi_{n}\left(\frac{1}{n} \Psi_{n}\right)\right)^{\prime} \otimes B\right]<1^{14}$.

Notice that the matrices $\Xi_{n}$ and $\left(\Phi_{n}\left(\frac{1}{n} \Psi_{n}\right)\right)^{\prime}$ have the following characteristics, the proof of which is in Appendix B.

\section{Lemma 1}

1. Eigenvalues of $\left(\Phi_{n}\left(\frac{1}{n} \Psi_{n}\right)\right)^{\prime}$ are all real and in $[0,1)$.

2. If $\lambda[B]>0, \lambda\left[\left(\Phi_{n}\left(\frac{1}{n} \Psi_{n}\right)\right)^{\prime} \otimes B\right]<\lambda\left[\Phi_{n}^{\prime} \otimes B\right]<\lambda\left[\Xi_{n} \otimes B\right]$.

3. $\lambda\left[\Xi_{n} \otimes B\right]=\lambda[B]$.

Hence, we find that the stability condition of the ODE (18) is solely $\lambda[B]<1$. First, if $\lambda[B] \leq 0$, the ODE is necessarily stable because $\lambda\left[\Xi_{n} \otimes B\right] \leq 0$ and $\lambda\left[\left(\Phi_{n}\left(\frac{1}{n} \Psi_{n}\right)\right)^{\prime} \otimes B\right] \leq 0$ by Lemma 1.1. Next, suppose $\lambda[B]>0 . \quad \lambda\left[\left(\Phi_{n}\left(\frac{1}{n} \Psi_{n}\right)\right)^{\prime} \otimes B\right]<\lambda\left[\Xi_{n} \otimes B\right]$ in Lemma 1.2 means that the stability of the $\operatorname{ODE}(18)$ is solely subject to whether $\lambda\left[\Xi_{n} \otimes B\right]<1$. As $\lambda\left[\Xi_{n} \otimes B\right]=\lambda[B]$, the stability condition is $\lambda[B]<1$.

\footnotetext{
${ }^{14}$ Note that if $n=1$, then $\Xi_{1}=1$ and $\frac{1}{1} \Psi_{1}=1$, and hence, the Jacobians are reduced to those under homogeneous learning, $(9,10)$.
} 
If agents recognize that no constant terms exist in the system (1) from the beginning, each type of agents will specify a PLM that excludes constant terms, instead of (12).

$$
y_{t}=c_{i} w_{i, t}+\varepsilon_{i, t}
$$

In this situation, the stability of the ODE (18) is subject to whether (22) has all negative real parts of eigenvalues. Then, the stability condition is $\lambda\left[\left(\Phi_{n}\left(\frac{1}{n} \Psi_{n}\right)\right)^{\prime} \otimes B\right]<1$.

Therefore, stability conditions of the HME are summarized as follows.

Proposition 2 In the system (1), if agents learn adaptively by specifying heterogeneous and misspecified models, a possible stable equilibrium is unique at the fixed point (19, 20) and deviates from the REE (8). If the equilibrium is called a Heterogeneous Misspecification Equilibrium (HME), the HME is locally stable if and only if:

1. under the PLM with constant terms (12), $\lambda[B]<1$;

2. under the PLM without constant terms (23), $\lambda\left[\left(\Phi_{n}\left(\frac{1}{n} \Psi_{n}\right)\right)^{\prime} \otimes B\right]<1$.

As $\lambda\left[\left(\Phi_{n}\left(\frac{1}{n} \Psi_{n}\right)\right)^{\prime} \otimes B\right]<\lambda[B]$ if $\lambda[B]>0$, Proposition 2 suggests that Corollary 1 continues to hold for heterogeneous learning.

Corollary 2 If agents recognize that no constant terms exist in the system (1) from the beginning and specify heterogeneous and misspecified PLMs without constant terms (23), stability conditions of the HME are less restrictive than otherwise. 
Compared with Proposition 1, Propostion 2 indicates that the effect of heterogeneity in learning depends upon whether agents include constant terms in their PLMs. If agents specify the PLM including constant terms, the stability condition under heterogeneous learning is equivalent to the condition under homogeneous learning. On the other hand, if agents specify the PLM without constant terms, the stability conditions under heterogeneous learning are less restrictive than the conditions under homogeneous learning. This is because $\lambda\left[\left(\Phi_{n}\left(\frac{1}{n} \Psi_{n}\right)\right)^{\prime} \otimes B\right]<\lambda\left[\Phi_{n}^{\prime} \otimes B\right]$ in Lemma 1.2, meaning that the real parts of the eigenvalues of coefficient matrix $B$ are allowed to have a wider range of values under heterogeneous learning than those permitted under homogeneous learning.

However, if we consider the real economy where there must exist some agents who specify PLMs without constant terms, the stability conditions under heterogeneous learning will almost surely be less restrictive than the conditions under homogeneous learning. For example, if at least one type of agents specify a PLM excluding constant terms, then $\Xi_{n}$ in the Jacobian (21) will be replaced with a matrix, e.g., $\Theta_{n}$, which has some elements equal to zero and the other elements equal to the counterparts of $\Xi_{n}$. Then, the stability condition is also replaced with $\lambda\left[\Theta_{n} \otimes B\right]<1$. As $\lambda\left[\Theta_{n} \otimes B\right]<\lambda\left[\Xi_{n} \otimes B\right]=\lambda[B]$, this stability condition is less restrictive than the condition under homogeneous learning. Thus, we can conclude:

Proposition 3 In the system (1), if agents learn adaptively by specifying heterogeneous and misspecified models, the stability conditions of the $\operatorname{HME}(19,20)$ will almost surely be less restrictive than the conditions of the REE (8) under the homogeneous and correctly specified learning rule. 
Therefore, if agents adopt the heterogeneous and misspecifed learning rules, the equilibrium will be more stable than the equilibrium under homogeneous learning. This suggests that the government may be able to choose between a wider range of policy instruments in the case of heterogeneous learning.

\subsection{Discussion}

The reason for the stabilization of the equilibrium under heterogeneous learning is because the aggregate forecasts $y_{t+1}^{e}$ become inactive against the exogenous variables $w_{t}$. Even if one of the exogenous variables, e.g., $w_{i, t}$, fluctuates, only agents of type $i$ will update their expectations given estimated parameters, while the other types of agents will not. Then, $y_{t+1}^{e}$ will not be revised as large as when all agents homogeneously respond to $w_{i, t}$. As a result, the economy tends to be stable when agents have inadequate and different information ${ }^{15}$.

Only if correlations between exogenous variables are high, the stability of the equilibrium under heterogeneous learning will be comparable with the stability of the equilibrium under homogeneous learning. If the correlations are high, the fluctuation of $w_{i, t}$ will lead to the fluctuations of other exogenous variables. Hence, even if agents have mutually different information, agents of different types tend to simultaneously update their expectations and make the equilibrium as unstable as the equilibrium under homogeneous learning. The effect of correlations between exogenous variables will be calibrated in the next section.

\footnotetext{
${ }^{15}$ Berardi (2007) numerically shows the possibility that the equilibrium may be stable under heterogeneous learning, but unstable uder homogeneous learning. We show a similar possibility analytically.
} 


\section{Example: New Keynesian Model}

In this section, we confirm our findings numerically by calibrating a basic New Keynesian $\operatorname{model}^{16}$.

$$
\left\{\begin{array}{l}
x_{t}=-\sigma\left(i_{t}-\pi_{t+1}^{e}\right)+x_{t+1}^{e}+g_{t} \\
\pi_{t}=\kappa x_{t}+\beta \pi_{t+1}^{e}+u_{t} \\
g_{t}=\mu g_{t-1}+\tilde{g}_{t} \\
u_{t}=\rho u_{t-1}+\tilde{u}_{t} .
\end{array}\right.
$$

The model has three endogenous variables: output gap $x_{t}$, inflation rate $\pi_{t}$, and nominal interest rate $i_{t}$. It has two exogenous variables $(n=2)$, a demand shock $g_{t} \backsim\left(0, \sigma_{g}^{2}\right)$ and a supply shock $u_{t} \backsim\left(0, \sigma_{u}^{2}\right)$, which follow disturbances $\tilde{g}_{t} \backsim\left(0, \sigma_{\tilde{g}}^{2}\right)$ and $\tilde{u}_{t} \backsim\left(0, \sigma_{\tilde{u}}^{2}\right)$. The correlation matrix of $g_{t}$ and $u_{t}$ is defined by $\Gamma_{2}=\left(\begin{array}{cc}1 & \rho_{g u} \\ \rho_{g u} & 1\end{array}\right)$, where $\rho_{g u}$ is a correlation between $g_{t}$ and $u_{t}$. This correlation is originally generated by the correlation between $\tilde{g}_{t}$ and $\tilde{u}_{t}$. We assume $\sigma, \kappa>0$, $0<\beta<1$, and $0<\mu, \rho<1$. To simplify our analysis, we assume that the persistence of the supply shock $u_{t}$ is no shorter than that of the demand shock $g_{t} ; 0<\mu \leq \rho<1$.

To close our model, a Taylor-type nominal interest rate rule is chosen by the central bank as follows.

$$
i_{t}=\phi_{\pi} \pi_{t}+\phi_{x} x_{t}
$$

$\phi_{\pi}, \phi_{x}$ are the policy parameters of the central bank, which are assumed to be positive.

This model is summarized by the representation of the system (1):

$$
\begin{aligned}
y_{t} & =\left(x_{t}, \pi_{t}\right)^{\prime}, w_{t}=\left(g_{t}, u_{t}\right)^{\prime}, v_{t}=\left(\tilde{g}_{t}, \tilde{u}_{t}\right)^{\prime}, \\
B & =\left(\begin{array}{cc}
1+\sigma \phi_{x} & \sigma \phi_{\pi} \\
-\kappa & 1
\end{array}\right)^{-1}\left(\begin{array}{cc}
1 & \sigma \\
0 & \beta
\end{array}\right), C=\left(\begin{array}{cc}
1+\sigma \phi_{x} & \sigma \phi_{\pi} \\
-\kappa & 1
\end{array}\right)^{-1}, \Phi_{2}=\left(\begin{array}{cc}
\mu & 0 \\
0 & \rho
\end{array}\right), \\
\Psi_{2} & =\frac{1}{2}\left(\begin{array}{cc}
\sigma_{g} & 0 \\
0 & \sigma_{u}
\end{array}\right)\left(\begin{array}{cc}
1 & \rho_{g u} \\
\rho_{g u} & 1
\end{array}\right)\left(\begin{array}{cc}
\sigma_{g} & 0 \\
0 & \sigma_{u}
\end{array}\right)^{-1} .
\end{aligned}
$$

\footnotetext{
${ }^{16}$ The structure and notations of our model follow those given by Evans and Honkapohja (2008). One difference is that $g_{t}$ and $u_{t}$ are allowed to correlate with each other. Our model is fundamentally equivalent with the models given by Woodford (1999) and Bullard and Mitra (2002).
} 
This has no effect on our results. Finally, we suppose that this model satisfies Assumptions 1 and 2 .

\subsection{Stability Conditions}

First, to consistently obtain stability conditions of equilibria under different learning rules, we provide a lemma.

Lemma 2 Define a $2 \times 2$ matrix $X$ whose eigenvalues are all real and in $[0,1]$. If $B=\left(\begin{array}{cc}1+\sigma \phi_{x} & \sigma \phi_{\pi} \\ -\kappa & 1\end{array}\right)^{-1}\left(\begin{array}{cc}1 & \sigma \\ 0 & \beta\end{array}\right)$, then

1. the real parts of eigenvalues of $X \otimes B-I_{4}$ are all negative if and only if

$$
\kappa\left(\phi_{\pi}-\lambda[X]\right)+\phi_{x}(1-\beta \lambda[X])>-\frac{(1-\lambda[X])(1-\beta \lambda[X])}{\sigma}
$$

2. as $\lambda[X]$ increases, the region $\left(\phi_{\pi}, \phi_{x}\right)$ that satisfies the above inequality will contract.

The proof is in Appendix C.

\subsubsection{Homogeneous Learning}

Under the correctly specified PLM with constant terms (4), the REE is locally stable if and only if the Jacobian (9) has negative real parts of eigenvalues. Eigenvalues of (9) are equivalent with those of $I_{2} \otimes B-I_{4}$. Following Lemma 2.1, $X=I_{2}$ and $\lambda\left[I_{2}\right]=1$. The stability condition is

$$
\kappa\left(\phi_{\pi}-1\right)+\phi_{x}(1-\beta)>0 .
$$


This is equivalent to the Taylor principle. This confirms the seminal result by Bullard and Mitra (2002) that the REE under a correctly specified PLM with constant terms is stable if and only if the central bank follows the Taylor principle.

If agents specify the PLM without constant terms (11), the sufficient and necessary condition for stability is that the Jacobian (10) has negative real parts of eigenvalues. Following Lemma 2.1, $X=\Phi_{2}^{\prime}$ and $\lambda\left[\Phi_{2}^{\prime}\right]=\rho$. The stability condition is

$$
\kappa\left(\phi_{\pi}-\rho\right)+\phi_{x}(1-\beta \rho)>-\frac{(1-\rho)(1-\beta \rho)}{\sigma}
$$

As $\lambda\left[\Phi_{2}^{\prime}\right]<\lambda\left[I_{2}\right]$ and Lemma 2.2 , the region $\left(\phi_{\pi}, \phi_{x}\right)$ to satisfy $(27)$ is larger than the region to satisfy (26). This result reinforces Corollary 1 that stability conditions are less restrictive if agents use the PLM without constant terms instead of the PLM with constant terms. Thus, if agents recognize no existence of constant terms in the system (1), the central bank does not have to respond to economic variables as actively as the Taylor principle dictates to ensure the learnability of the REE.

\subsubsection{Heterogeneous Learning}

Next, we consider the existence of two types of agents. Type 1 can observe the demand shock $g_{t}$ but cannot observe the supply shock $u_{t}$, while Type 2 can observe $u_{t}$ but cannot observe $g_{t}$. The populations of both types are set at $\frac{1}{2}$.

As is shown in Proposition 2.1, if agents specify the heterogeneous and underparameterized PLMs with constant terms, (12), the sufficient and necessary condition for the HME to be stable is the same as $(26)$.

Under the heterogeneous and underparameterized PLMs without constant terms, (23), the 
HME is locally stable if and only if the Jacobian (22) has negative real parts of eigenvalues. Following Lemma 2, $X=\left(\Phi_{2}\left(\frac{1}{2} \Psi_{2}\right)\right)^{\prime}$ and

$$
\lambda\left[\left(\Phi_{2}\left(\frac{1}{2} \Psi_{2}\right)\right)^{\prime}\right]=\frac{1}{4}(\mu+\rho)+\frac{1}{4} \sqrt{(\mu+\rho)^{2}-4 \mu \rho\left(1-\rho_{g u}^{2}\right)},
$$

which is in $(0, \rho)$. The stability condition is

$$
\begin{aligned}
\kappa\left(\phi_{\pi}-\lambda\left[\left(\Phi_{2}\left(\frac{1}{2} \Psi_{2}\right)\right)^{\prime}\right]\right)+\phi_{x} & \left(1-\beta \lambda\left[\left(\Phi_{2}\left(\frac{1}{2} \Psi_{2}\right)\right)^{\prime}\right]\right) \\
> & -\frac{\left(1-\lambda\left[\left(\Phi_{2}\left(\frac{1}{2} \Psi_{2}\right)\right)^{\prime}\right]\right)\left(1-\beta \lambda\left[\left(\Phi_{2}\left(\frac{1}{2} \Psi_{2}\right)\right)^{\prime}\right]\right)}{\sigma} .
\end{aligned}
$$

As $\lambda\left[\left(\Phi_{2}\left(\frac{1}{2} \Psi_{2}\right)\right)^{\prime}\right]<\lambda\left[\Phi_{2}^{\prime}\right]=\rho$ and Lemma 2.2 , the stability conditions of the HME are less restrictive than the conditions of the REE, (27). This confirms Proposition 3. Therefore, if agents choose heterogeneous and misspecified learning rules, the central bank may be less active in ensuring the learnability of the equilibrium.

\subsubsection{Impact of the correlation of demand and supply shocks}

Furthermore, in this basic model, we can clarify the relationship between the correlation of demand and supply shocks, $\rho_{g u}$, and the stability conditions under heterogeneous learning.

Proposition 4 In the basic New Keynesian model (24) with the Taylor-type nominal interest rate rule (25), as the correlation of demand and supply shocks, $\rho_{g u}$, decreases, stability conditions of the HME become further less restrictive than the Taylor principle.

The proof is in Appendix D.

This proposition is intuitive. If exogenous variables are not correlated with one another, they are idiosyncratic. This situation means an increase in heterogeneity in the sense that 
agents specify heterogeneous PLMs. As a result, stability conditions become less restrictive. On the other hand, for example, if $\rho_{g u}=1$ and $\mu=\rho$, then $(28)$ gives $\lambda\left[\left(\Phi_{2}\left(\frac{1}{2} \Psi_{2}\right)\right)^{\prime}\right]=\rho$; the conditions become equivalent to those under homogeneous learning, (27). This is because if $\rho_{g u}=1$ and $\mu=\rho$, both types of agents act as if they observe and respond to common variables. As a result, the stability conditions are reduced to those under homogeneous learning.

\subsection{Calibrations}

We confirm numerically the previous results by assuming $\kappa=0.125, \beta=0.99, \sigma=1, \mu=0.8$, and $\rho=0.9$. Similar calibrations are shown by Evans and Honkapohja (2001, chapters 4 and $8)$.

\subsubsection{Stability Conditions}

First, we show that stability conditions of the HME are less restrictive than the conditions of the REE under a homogeneous and correctly specified learning rule. Figure 1 shows the regions of values of the policy parameter $\left(\phi_{\pi}, \phi_{x}\right)$ to satisfy stability conditions. In the first quadrant $\left(\phi_{\pi}, \phi_{x}>0\right)$, the region under heterogeneous learning perfectly encompasses the region under homogeneous learning, irrespective of the value of the correlation $\rho_{g u}$. In addition, as $\rho_{g u}$ decreases, the region under heterogeneous learning expands. If $\rho_{g u}<0.7$, this region almost encompasses the first quadrant, meaning that almost any values of $\left(\phi_{\pi}, \phi_{x}\right)$ satisfy the stability conditions $^{17}$. Hence, if agents form heterogeneous expectations based on their observable shocks, the central bank might not have to address the learnability of the equilibrium.

\footnotetext{
${ }^{17}$ Cover et al. (2006) estimate the correlation between demand and supply shocks in a simple AD-AS model to be approximately 0.7 using US data. Enders and Hurn (2007), using Australian data, estimate the correlation to be 0.73 .
} 


\subsubsection{Parameters Updating}

Next, we calibrate the dynamics of parameter estimates $a_{t} \equiv\left(\begin{array}{c}a_{x, t} \\ a_{\pi, t}\end{array}\right)$ and $c_{t} \equiv\left(\begin{array}{cc}c_{x g, t} & c_{x u, t} \\ c_{\pi g, t} & c_{\pi u, t}\end{array}\right)$ under recursive least squares and find differences between homogeneous and heterogeneous learning. We set the variance-covariance matrix of $g_{t}$ and $u_{t}$ to be $\left(\begin{array}{cc}0.69 & 0.71 \\ 0.71 & 1.32\end{array}\right)$, which corresponds to the variance-covariance matrix of $\tilde{g}_{t}$ and $\tilde{u}_{t}$ by $\left(\begin{array}{cc}0.25 & 0.2 \\ 0.2 & 0.25\end{array}\right)$. The correlation of $g_{t}$ and $u_{t}$, $\rho_{g u}$, is 0.75 . To simplify our analysis, we assume $\phi_{x}=0$. We set the initial beliefs of agents on the parameters in their PLMs to be unity, that is, $a_{0}=\left(\begin{array}{l}1 \\ 1\end{array}\right)$ and $c_{0}=\left(\begin{array}{ll}1 & 1 \\ 1 & 1\end{array}\right){ }^{18}$.

Figure 2 shows the dynamics of the estimates when the central bank chooses an active policy $\phi_{\pi}=5$, which satisfies the stability condition under both homogeneous and heterogeneous learning rules with constant terms, (26). Solid lines (blue) represent the transitions of estimates under homogeneous learning. Dotted (black) and dashed (red) lines represent the transitions of estimates of agents of Types 1 and 2 under heterogeneous learning, respectively. Under heterogeneous learning, $a_{t}$ is estimated by both types of agents, whereas $\left(c_{x g}, c_{\pi g}\right)$ and $\left(c_{x u}, c_{\pi u}\right)$ are respectively estimated by agents of Type 1 and 2 . We confirm that all estimates converge to the fixed points and that the HME under heterogeneous learning diverts from the REE under homogeneous learning.

Note that even if agents exclude constant terms from their learning rules, this active policy continues to ensure the learnability of the REE and the HME (Corollaries 1 and 2). One may consider that the dynamics of $c_{t}$ under learning rules without constant terms are almost the same as those under the rules with constant terms. Hence, those results are abbreviated.

Next, Figure 3 shows the dynamics of the estimates when the central bank chooses a passive

\footnotetext{
${ }^{18}$ In addition, we set the initial beliefs on $R_{i, t}=t^{-1} \sum_{s=1}^{t} z_{i, t} z_{i, t}^{\prime}$ where $z_{i, t}^{\prime}=\left(1, w_{i, t}\right)$, which are only shown in Appendix A, to be equal to its unconditional expectation, $E z_{i} z_{i}^{\prime}$.
} 
policy $\phi_{\pi}=0.5$, which does not satisfy the stability condition under a homogeneous learning rule without constant terms, (27), but satisfies the condition under heterogeneous learning rules without constant terms, (29). Then, all estimates of $a$ explode irrespective of heterogeneity in expectations. On the other hand, $\left(c_{x u}, c_{\pi u}\right)$ explode under homogeneous learning, while all estimates of $c$ converge to the fixed point under heterogeneous learning. Thus, this figure proves Proposition 3 that stability conditions under heterogeneous learning are less restrictive than those under homogeneous learning. In addition, under heterogeneous learning, the HME is stable if constant terms are excluded from the PLMs.

Furthermore, we will figure out the relationship between the correlation of demand and supply shocks and stability conditions under adaptive learning. We assume a further passive policy $\phi_{\pi}=0.1$, which satisfies the stability conditions under neither homogeneous learning, (27), nor heterogeneous learning, (29). Figure 4 shows the dynamics under $\phi_{\pi}=0.1$. Even under heterogeneous learning, several estimates of $c$ also explode. Then, the HME as well as the REE is unstable.

On the other hand, if $g_{t}$ and $u_{t}$ are not correlated, that is, $\rho_{g u}=0$, then the HME becomes stable. Figure 5 shows the dynamics under $\phi_{\pi}=0.1$ and $\rho_{g u}=0$, which do not satisfy the stability condition under a homogeneous learning rule without constant terms, (27), but satisfies the condition under heterogeneous learning rules without constant terms, (29). Estimates of $c$ under homogeneous learning keep exploding, while estimates of $c$ under heterogeneous learning converge to the fixed point. This figure reinforces Proposition 4 that as $\rho_{g u}$ decreases, stability conditions under heterogeneous learning become further less restrictive.

Therefore, in a basic NK model, if agents conduct heterogeneous learning, and further, if 
agents specify PLMs without constant terms, then the learnability of the equilibrium will hold with almost any values of policy parameters of the central bank.

\section{Conclusions}

This paper has investigated characteristics of equilibrium and its stability conditions under heterogeneous learning in an environment in which each agent has an imperfect and different information set about exogenous variables and forms an underparameterized and heterogeneous belief of the structure of the economy.

The findings of the paper are as follows. First, under heterogeneous learning, the economy converges to a Heterogeneous Misspecification Equilibrium (HME) that deviates from a rational expectations equilibrium (REE). Next, the stability conditions of the HME are less restrictive than conditions of the REE. For example, in a basic NK model with a Taylor-type monetary policy rule, the central bank is allowed to choose a wider range of values of policy parameters to ensure economic stability under heterogeneous learning than those that are permitted under homogeneous learning. In particular, if the correlation between demand and supply shocks is low, the central bank might not have to address the learnability of the equilibrium.

In future works, we could examine the relationship between the degree of heterogeneity in adaptive learning and the stability conditions of the equilibrium. Most studies, including the present study, examine conditions for the learnability of the equilibrium in situations in which the degree of heterogeneity is given. We may be able to clarify whether and how the learnability of the equilibrium is affected by a change in the degree of heterogeneity in learning. If the number of exogenous variables increases so that the degree of the heterogeneity among 
agents' learning increases, stability conditions of the equilibrium might be further loosened.

\section{Appendix}

\section{A Derivation of Definition 1}

We follow the methodology of Evans and Honkapohja (2001, chapter 13) to obtain the ordinary differential equations under misspecified PLMs.

Agent $i$ forms $y_{t+1}^{e}$ by real-time learning with the misspecified PLM (12) and the information set $\left\{y_{s}, w_{i, s}\right\}_{s=1}^{t}$. The coefficient parameters, $\phi_{i, t}^{\prime}=\left(a_{i, t}, c_{i, t}\right)$, are given by the least-squares projection of $y_{t}$ on $z_{i, t}^{\prime}=\left(1, w_{i, t}\right)$. That is, $\phi_{i, t}^{\prime}$ must satisfy

$$
E z_{i, t}\left(y_{t}-\phi_{i, t}^{\prime} z_{i, t}\right)=\mathbf{0}
$$

and the updating rule of $\phi_{i, t}^{\prime}$ is shown as the recursive least-squares representation:

$$
\begin{aligned}
\phi_{i, t} & =\phi_{i, t-1}+t^{-1} R_{i, t}^{-1} z_{i, t-1}\left(y_{t-1}-\phi_{i, t-1}^{\prime} z_{i, t-1}\right)^{\prime} \\
R_{i, t} & =R_{i, t-1}+t^{-1}\left(z_{i, t-1} z_{i, t-1}^{\prime}-R_{i, t-1}\right)
\end{aligned}
$$

where $R_{i, t}=t^{-1} \sum_{s=1}^{t} z_{i, t} z_{i, t}^{\prime}$. The operator $E$ denotes the expectation of variables, for $\phi_{i}$ fixed, taken over the invariant distributions of the exogenous variables $w_{t}$. See Evans and Honkapohja (2001, chapter 10.3) for the detail of obtaining recursive least squares equations to satisfy the orthogonality condition in the models with contemporaneous expectations.

The actual law of motion under the real-time updating is given (16). By substituting (16) into (30), the stochastic recursive algorithm (SRA) for $\phi_{i, t}$ is obtained as follows.

$\left.\phi_{i, t}=\phi_{i, t-1}+t^{-1} R_{i, t}^{-1} z_{i, t-1}\left(1, w_{1, t-1}, \ldots, w_{n, t-1}\right)\left[\begin{array}{llll}D_{0, t-1} & D_{1, t-1} & \cdots & D_{n, t-1}\end{array}\right)-\left(\begin{array}{ll}a_{i, t-1} & c_{i, t-1}^{+}\end{array}\right)\right]^{\prime}$, 
where $c_{i, t}^{+}$is a $m \times n$ matrix $\left(\mathbf{0}, \ldots, \mathbf{0}, c_{i, t}, \mathbf{0}, \ldots, \mathbf{0}\right)$, which corresponds to $c_{t} \equiv\left(c_{1, t}, c_{2, t}, \ldots, c_{n, t}\right)$ in which the columns except for $c_{i, t}$ are zero, and $D_{0, t} \equiv \frac{1}{n} B\left(I_{m}, \ldots, I_{m}\right) a_{t}$ and $D_{i, t} \equiv \frac{1}{n} B c_{i, t} \varphi_{i}+C_{i}$ for $i \in\{1, \ldots, n\}$.

The convergence of the SRA is studied as the stochastic approximation approach by Marcet and Sargent (1989). This is also introduced by Evans and Honkapohja (2001, chapter 6). By letting $E z_{i} z_{j}^{\prime}=\lim _{t \rightarrow \infty} E z_{i, t} z_{j, t}^{\prime}$ for any $i, j \in\{1, \ldots, n\}$,

$$
\begin{aligned}
& E R_{i}^{-1} z_{i, t-1}\left(1, w_{1, t-1}, \ldots, w_{n, t-1}\right)\left[\left(\begin{array}{cccc}
D_{0} & D_{1} & \cdots & D_{n}
\end{array}\right)-\left(\begin{array}{ll}
a_{i} & c_{i}^{+}
\end{array}\right)\right]^{\prime} \\
& =R_{i}^{-1}\left(\left(E z_{i, t-1} z_{i, t-1}^{\prime}\right)\left[\left(\begin{array}{cc}
D_{0} & D_{i}
\end{array}\right)-\left(\begin{array}{ll}
a_{i} & c_{i}
\end{array}\right)\right]^{\prime}+E\left(\begin{array}{c}
1 \\
w_{i, t-1}
\end{array}\right)\left(\sum_{j=1}^{n} w_{j, t-1} D_{j}^{\prime}-w_{i, t-1} D_{i}^{\prime}\right)\right) \\
& =R_{i}^{-1}\left(\begin{array}{c}
\left.\left(E z_{i, t-1} z_{i, t-1}^{\prime}\right)\left[\begin{array}{cc}
D_{0} & D_{i}
\end{array}\right)-\left(\begin{array}{cc}
a_{i} & c_{i}
\end{array}\right)\right]^{\prime} \\
\quad+\left(\begin{array}{c}
\mathbf{0} \\
\sum_{j=1}^{n}\left(E w_{i, t-1} w_{j, t-1}\right) D_{j}^{\prime}-\left(E w_{i, t-1} w_{i, t-1}\right) D_{i}^{\prime}
\end{array}\right)
\end{array}\right) \\
& =R_{i}^{-1}\left(E z_{i, t-1} z_{i, t-1}^{\prime}\right)\left(\begin{array}{c}
\left.\left[\begin{array}{cc}
D_{0} & D_{i}
\end{array}\right)-\left(\begin{array}{cc}
a_{i} & c_{i}
\end{array}\right)\right]^{\prime} \\
\quad+\left(\begin{array}{c}
\mathbf{0} \\
\sum_{j=1}^{n}\left(E w_{i, t-1} w_{i, t-1}\right)^{-1}\left(E w_{i, t-1} w_{j, t-1}\right) D_{j}^{\prime}-D_{i}^{\prime}
\end{array}\right)
\end{array}\right) .
\end{aligned}
$$

Hence, the associated ordinary differential equation (ODE) for the SRA is obtained as

$$
\begin{aligned}
\frac{d \phi_{i}}{d \tau} & =R_{i}^{-1}\left(E z_{i} z_{i}^{\prime}\right)\left(T_{i}(a, c)-\left(a_{i}, c_{i}\right)\right)^{\prime} \\
\frac{d R_{i}}{d \tau} & =E z_{i} z_{i}^{\prime}-R_{i}
\end{aligned}
$$

where

$$
T_{i}(a, c)=\left(T_{i, a}(a), T_{i, c}(c)\right)=\left(D_{0}, \sum_{j=1}^{n} D_{j} \Omega_{i j} \Omega_{i i}^{-1}\right) .
$$

$\Omega_{i j}$ is a covariance of $w_{i}$ and $w_{j}$; then, $\Omega_{i j}=\sigma_{i i} \rho_{i j} \sigma_{j j}$ and $\Omega_{i j}=\Omega_{j i}$. Since $R_{i}$ and $E z_{i} z_{i}^{\prime}$ in (32) are asymptotically equal, $R_{i}^{-1}\left(E z_{i} z_{i}^{\prime}\right)$ in $(31)$ globally converges to unity. Hence, the stability 
of the ODE for $\phi_{i}^{\prime}=\left(a_{i}, c_{i}\right)$ is determined by a smaller differential equation.

$$
\frac{d \phi_{i}}{d \tau}=T_{i}(a, c)-\left(a_{i}, c_{i}\right)
$$

After obtaining the ODEs of all agents, we can summarize the dynamics of the parameter estimates of all types, $\left\{\phi_{i}\right\}_{i=1}^{n}$, as the following ODE of $a=\left(a_{1}^{\prime}, \ldots, a_{n}^{\prime}\right)^{\prime}$ and $c=\left(c_{1}, \ldots, c_{n}\right)$,

$$
\begin{aligned}
& \frac{d a}{d \tau}=T_{a}(a)-a \\
& \frac{d c}{d \tau}=T_{c}(c)-c
\end{aligned}
$$

where

$$
\begin{aligned}
& T_{a}(a) \equiv\left(\begin{array}{c}
T_{1, a}(a) \\
\vdots \\
T_{n, a}(a)
\end{array}\right)=\left(\Xi_{n} \otimes B\right) a \\
& T_{c}(c) \equiv\left(T_{1, c}(c), \ldots, T_{n, c}(c)\right)=\left(\frac{1}{n} B c \Phi_{n}+C\right) \Psi_{n}, \\
& \Psi_{n} \equiv\left(\begin{array}{cccc}
1 & \Omega_{12} \Omega_{22}^{-1} & \ldots & \Omega_{1 n} \Omega_{n n}^{-1} \\
\Omega_{21} \Omega_{11}^{-1} & 1 & \cdots & \Omega_{2 n} \Omega_{n n}^{-1} \\
\vdots & \vdots & \ddots & \vdots \\
\Omega_{n 1} \Omega_{11}^{-1} & \Omega_{n 2} \Omega_{22}^{-1} & \cdots & 1
\end{array}\right)=\operatorname{diag}\left(\sigma_{i i}\right) \Gamma_{n} \operatorname{diag}\left(\sigma_{i i}\right)^{-1} .
\end{aligned}
$$

These results are summarized in Definition 1.

\section{B Proof of Lemma 1}

In order to prove Lemma 1, we initially provide two mathematical lemmas.

Lemma 3 If any real matrix $X$ is positive (resp., negative) definite, and if any real matrix $Y$ is of the same dimension and nonnegative (nonpositive) definite, then eigenvalues of XY are all real and nonnegative (nonpositive). 
Proof. If $\gamma$ is any eigenvalue of $X Y$, there exists a non-null vector $w$ such that $X Y w=\gamma w$. Write $w=x+y i$ and $\gamma=\alpha+\beta i$, where $x$ and $y$ are real vectors, and $\alpha$ and $\beta$ are real scalars. Write also the complex conjugate of $w$ as $\bar{w}=x-y i$. Premultiply both sides by $\bar{w}^{\prime} X^{-1}$,

$$
\bar{w}^{\prime} X^{-1} X Y w=\bar{w}^{\prime} Y w=\gamma \bar{w}^{\prime} X^{-1} w .
$$

Expanding (33),

$$
\begin{aligned}
\bar{w}^{\prime} Y w & =\left(x^{\prime} Y x+y^{\prime} Y y\right)+i\left(x^{\prime} Y y-y^{\prime} Y x\right) \\
& =x^{\prime} Y x+y^{\prime} Y y
\end{aligned}
$$

and

$$
\begin{aligned}
\gamma \bar{w}^{\prime} X^{-1} w & =(\alpha+\beta i)\left(\left(x^{\prime} X^{-1} x+y^{\prime} X^{-1} y\right)+i\left(x^{\prime} X^{-1} y-y^{\prime} X^{-1} x\right)\right) \\
& =\alpha\left(x^{\prime} X^{-1} x+y^{\prime} X^{-1} y\right)+i \beta\left(x^{\prime} X^{-1} x+y^{\prime} X^{-1} y\right)
\end{aligned}
$$

where $x^{\prime} Y y-y^{\prime} Y x=\mathbf{0}$ and $x^{\prime} X^{-1} y-y^{\prime} X^{-1} x=\mathbf{0}$. Further, equating real and imaginary parts of (34) and (35),

$$
\begin{aligned}
x^{\prime} Y x+y^{\prime} Y y & =\alpha\left(x^{\prime} X^{-1} x+y^{\prime} X^{-1} y\right) \\
\mathbf{0} & =\beta\left(x^{\prime} X^{-1} x+y^{\prime} X^{-1} y\right) .
\end{aligned}
$$

If $X$ is positive (negative) definite, and if $Y$ is nonnegative (nonpositive) definite, then $x^{\prime} X^{-1} x+$ $y^{\prime} X^{-1} y>(<) \mathbf{0}$ and $x^{\prime} Y x+y^{\prime} Y y \geq(\leq) \mathbf{0}$. To satisfy $(37), \beta=\mathbf{0} ;$ then $\gamma$ is real. To satisfy (36), $\alpha \geq(\leq) \mathbf{0}$; then $\gamma$ is nonnegative (nonpositive). Therefore, eigenvalues of $X Y$ are all real and nonnegative (nonpositive). (To prove this lemma, we referred to the Proof of Lemma 3 and Theorem 1 in Kenneth J. Arrow and Maurice McManus, "A Note on Dynamic Stability," 
Econometrica, 1958, 26, 448-454.)

Lemma $40 \leq \lambda\left[\left(\Phi_{n}\left(\frac{1}{n} \Psi_{n}\right)\right)^{\prime}\right]<\lambda\left[\Phi_{n}^{\prime}\right]<1$

Proof. Because the correlation matrix $\Gamma_{n}$ is a nonnegative definite matrix, the eigenvalues of $\frac{1}{n} \Gamma_{n}$ are all nonnegative, and at least one of them is positive. Then, $\lambda\left[\frac{1}{n} \Gamma_{n}\right]>0$. The diagonal elements of $\frac{1}{n} \Gamma_{n}$ are all $\frac{1}{n}$, so $\operatorname{tr}\left(\frac{1}{n} \Gamma_{n}\right)=1$. Then $\lambda\left[\frac{1}{n} \Gamma_{n}\right]<1$. As a result,

$$
0<\lambda\left[\frac{1}{n} \Gamma_{n}\right]<1
$$

Next, as $\Phi_{n} \geq \mathbf{0}$ and $\frac{1}{n} \Gamma_{n} \geq \mathbf{0}$ from Assumption $2, \mathbf{0} \leq \Phi_{n}\left(\frac{1}{n} \Gamma_{n}\right) \leq \lambda\left[\Phi_{n}\right]\left(\frac{1}{n} \Gamma_{n}\right)$. By the PerronFrobenius Theorem (see Peter Berck and Knut Sydsaeter, Economists' Mathematical Manual Second Edition, Springer-Verlag, 1993, p.110), this inequality means $0 \leq \lambda\left[\Phi_{n}\left(\frac{1}{n} \Gamma_{n}\right)\right] \leq$ $\lambda\left[\lambda\left[\Phi_{n}\right]\left(\frac{1}{n} \Gamma_{n}\right)\right]=\lambda\left[\Phi_{n}\right] \lambda\left[\frac{1}{n} \Gamma_{n}\right]$. By $(38), \lambda\left[\Phi_{n}\right] \lambda\left[\frac{1}{n} \Gamma_{n}\right]<\lambda\left[\Phi_{n}\right]<1$. As a result

$$
0 \leq \lambda\left[\Phi_{n}\left(\frac{1}{n} \Gamma_{n}\right)\right]<\lambda\left[\Phi_{n}\right]<1
$$

Finally, Notice that eigenvalues of $\Phi_{n}\left(\frac{1}{n} \Psi_{n}\right)$ are equivalent to those of $\Phi_{n}\left(\frac{1}{n} \Gamma_{n}\right)$ because $\Phi_{n}\left(\frac{1}{n} \Psi_{n}\right)=\operatorname{diag}\left(\sigma_{i i}\right)\left(\Phi_{n}\left(\frac{1}{n} \Gamma_{n}\right)\right) \operatorname{diag}\left(\sigma_{i i}\right)^{-1}$. Therefore, (39) results in

$$
0 \leq \lambda\left[\left(\Phi_{n}\left(\frac{1}{n} \Psi_{n}\right)\right)^{\prime}\right]<\lambda\left[\Phi_{n}^{\prime}\right]<1
$$

With Lemmas 3 and 4, Lemma 1 is obtained as follows. First, since $\Phi_{n}$ is a positive definite matrix and $\frac{1}{n} \Psi_{n}$ is a nonnegative definite matrix, eigenvalues of $\Phi_{n}\left(\frac{1}{n} \Psi_{n}\right)$ and $\left(\Phi_{n}\left(\frac{1}{n} \Psi_{n}\right)\right)^{\prime}$ are 
found to be all real and nonnegative by Lemma 3. This result and Lemma 4 lead to Lemma 1.1. Next, $\lambda\left[\Xi_{n}\right]=1$; then Lemma 4 leads to $\lambda\left[\left(\Phi_{n}\left(\frac{1}{n} \Psi_{n}\right)\right)^{\prime}\right]<\lambda\left[\Phi_{n}^{\prime}\right]<\lambda\left[\Xi_{n}\right]=1$. In addition, eigenvalues of $\left(\Phi_{n}\left(\frac{1}{n} \Psi_{n}\right)\right)^{\prime}, \Phi_{n}^{\prime}$, and $\Xi_{n}$ are all real and nonnegative. Thus, their Kronecker products with matrix $B$ that suffices $\lambda[B]>0$ hold the same inequalities as Lemma 1.2. Finally, Lemma 1.3 is trivial.

\section{Proof of Lemma 2}

Define the eigenvalues of $X$ as $0 \leq \chi_{i} \leq 1 \quad(i=1, \ldots, n)$ and the eigenvalues of $B$ as $\delta_{j}$ $(j=1, \ldots, m)$; then the eigenvalues of $X \otimes B$ are given by $\chi_{i} \delta_{j}$ for any $i, j$. First, if $\lambda[B] \leq 0$, the real parts of eigenvalues of $X \otimes B-I_{4}$ are necessarily all negative. Next, suppose $\lambda[B]>0$. Since $\chi_{i} \geq 0, \lambda[X \otimes B]=\lambda[X] \lambda[B]=\lambda[\lambda[X] B]$; then $\lambda\left[X \otimes B-I_{m n}\right]=\lambda\left[\lambda[X] B-I_{m}\right]$. This means that eigenvalues of $X \otimes B-I_{m n}$ are all negative if and only if eigenvalues of $\lambda[X] B-I_{m}$ have all negative real parts. The characteristic equation of $\lambda[X] B-I_{m}$ is obtained by

$$
\begin{aligned}
q(x) & =x^{2}+p_{1} x+p_{0}, \\
p_{0} & =\frac{(1-\lambda[X])(1-\beta \lambda[X])+\sigma\left(\kappa\left(\phi_{\pi}-\lambda[X]\right)+\phi_{x}(1-\beta \lambda[X])\right)}{1+\kappa \sigma \phi_{\pi}+\sigma \phi_{x}}, \\
p_{1} & =\frac{(1-\lambda[X])+(1-\beta \lambda[X])+\kappa \sigma\left(2 \phi_{\pi}-\lambda[X]\right)+\sigma \phi_{x}(2-\beta \lambda[X])}{1+\kappa \sigma \phi_{\pi}+\sigma \phi_{x}},
\end{aligned}
$$

where $p_{1}=p_{0}+\frac{(1-\beta \lambda[X])+\sigma\left(\kappa \phi_{\pi}+\phi_{x}\right)}{1+\kappa \sigma \phi_{\pi}+\sigma \phi_{x}}$, that is, $p_{1}>p_{0}$. Proposition 10.3 of Evans and Honkapohja (2001) implies that the equilibrium is locally stable under adaptive learning if and only if the real parts of all eigenvalues are negative. According to the Routh Theorem (see Alpha 
C. Chiang, Fundamental Methods of Mathematical Economics: Second Edition, McGraw-Hill, 1974), eigenvalues of $\lambda[X] B-I_{m}$ have all negative real parts if and only if $\left|p_{1}\right|$ and $\left|\begin{array}{cc}p_{1} & 0 \\ 1 & p_{0}\end{array}\right|$ are all positive, that is, $p_{1}>0$ and $p_{1} p_{0}>0$. As $p_{1}>p_{0}$, those conditions are reduced to $p_{0}>0$, which is

$$
\kappa\left(\phi_{\pi}-\lambda[X]\right)+\phi_{x}(1-\beta \lambda[X])>-\frac{(1-\lambda[X])(1-\beta \lambda[X])}{\sigma}
$$

As $0 \leq \lambda[X] \leq 1$, the second part of the Lemma is easily obtained.

\section{Proof of Proposition 4}

Equation (28) gives $\frac{d}{d \rho_{g u}} \lambda\left[\left(\Phi_{2}\left(\frac{1}{2} \Psi_{2}\right)\right)^{\prime}\right]>0$. Furthermore, note Lemma 2.2. Therefore, as $\rho_{g u}$ decreases, $\lambda\left[\left(\Phi_{2}\left(\frac{1}{2} \Psi_{2}\right)\right)^{\prime}\right]$ also decreases, and hence the region $\left(\phi_{\pi}, \phi_{x}\right)$ that satisfies the condition for stability will expand.

\section{References}

AdAm, K. (2005): "Experimental Evidence on the Persistence of Output and Inflation," Economic Journal, 117, 603-636.

Berardi, M. (2007): "Heterogeneity and Misspecifications in Learning," Journal of Economic Dynamics and Control, 31, 3203-3227. 
(2009a): "Heterogeneous Expectations, Sunspot Equilibria and their Fragility," Economics Letters, 105, 276-279.

_ (2009b): "Monetary Policy with Heterogeneous and Misspecified Expectations," Journal of Money, Credit, and Banking, 41, 79-100.

Branch, W. A. (2004): "Restricted Perceptions Equilibria and Learning in Macroeconomics," in Post Walrasian Macroeconomics: Beyond the Dynamic Stochastic General Equilibrium Model, ed. by D. Colander, New York: Cambridge University Press, 135-160.

Branch, W. A. And G. W. Evans (2006): "Intrinsic Heterogeneity in Expectation Formation," Journal of Economic Theory, 127, 264-295.

(2007): "Model Uncertainty and Endogenous Volatility," Review of Economic Dynamics, 10, 207-237.

Branch, W. A. And B. McGough (2004): "Multiple Equilibria in Heterogeneous Expectations Models," Contributions to Macroeconomics, 4, Article 12.

_ (2009): "A New Keynesian Model with Heterogeneous Expectations," Journal of Economic Dynamics and Control, 33, 1036-1051.

Brock, W. A. And C. H. Hommes (1997): "A Rational Route to Randomeness," Econometrica, 65, 1059-1095.

Bullard, J. AND K. Mitra (2002): "Learning about Monetary Policy Rules," Journal of Monetary Economics, 49, 1105-1129. 
Cover, J. P., W. Enders, And C. J. Hueng (2006): "Using the Aggregate DemandAggregate Supply Model to Identify Structural Demand-Side and Supply Side Shocks: Results Using a Bivariate VAR," Journal of Money, Credit, and Banking, 38, 777-790.

Duffy, J. And W. XiaO (2007): "Instability of Sunspot Equilibria in Real Business Cycle Models under Adaptive Learning," Journal of Monetary Economics, 54, 879-903.

Enders, W. And S. Hurn (2007): "Identifying Aggregate Demand and Supply Shocks in a Small Open Economy," Oxford Economic Papers, 59, 411-429.

Evans, G. W. And S. Honkapohja (1996): "Least Squares Learning with Heterogeneous Expectations," Economics Letters, 53, 197-201.

(2001): Learning and Expectations in Macroeconomics, Princeton University Press.

(2008): "Expectations, Learning and Monetary Policy: An Overview of Recent Research," Centre for Dynamic Macroeconomic Analysis Working Paper Series, 08/02.

Evans, G. W., S. Honkapohja, And R. Marimon (2001): "Convergence in Monetary Inflation Models with Heterogeneous Learning Rules," Macroeconomic Dynamics, 5, 1-31.

Evans, G. W. And B. McGough (2005): "Monetary Policy, Indeterminacy and Learning," Journal of Economic Dynamics and Control, 29, 1809-1840.

Giannitsarou, C. (2003): "Heterogeneous Learning," Review of Economic Dynamics, 6, 885906.

Guse, E. A. (2005): "Stability Properties for Learning with Heterogeneous Expectations and Multiple Equilibria," Journal of Economic Dynamics and Control, 29, 1623-1642. 
Hommes, C. H. And G. Sorger (1998): "Consistent Expectations Equilibria," Macroeconomic Dynamics, 2, 287-321.

Honkapohja, S. And K. Mitra (2005): "Performance of Monetary Policy with Internal Central Bank Forecasting," Journal of Economic Dynamics and Control, 29, 627-658.

(2006): "Learning Stability in Economies with Heterogeneous Agents," Review of Economic Dynamics, 9, 284-309.

Marcet, A. And T. J. Sargent (1989): "Convergence of Least-Squares Learning Mechanisms in Self-Referential Linear Stochastic Models," Journal of Economic Theory, 48, 337368.

Woodford, M. (1999): "Optimal Monetary Policy Inertia," Manchester School, Supplement, $61,1-35$.

The English in this document has been checked by at least two professional editors, both native speakers of English. For a certificate, please see:

http://www.textcheck.com/certificate/JzURHW 
Figure 1 Stability Conditions on the Policy Parameters $\left(\phi_{\pi}, \phi_{x}\right)$ and the Effect of Correlation ( $\rho g u)$

(a) Homogeneous Learning

$$
(n=1)
$$

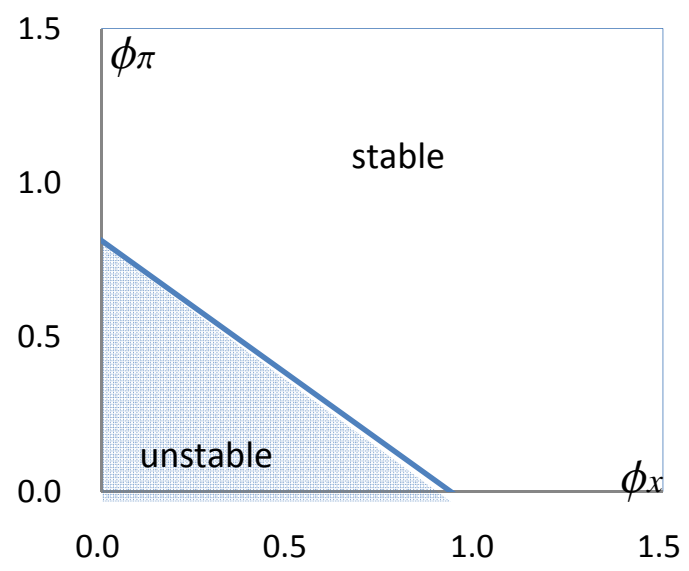

(c) Heterogeneous Learning

$$
(n=2, \rho g u=0.9)
$$

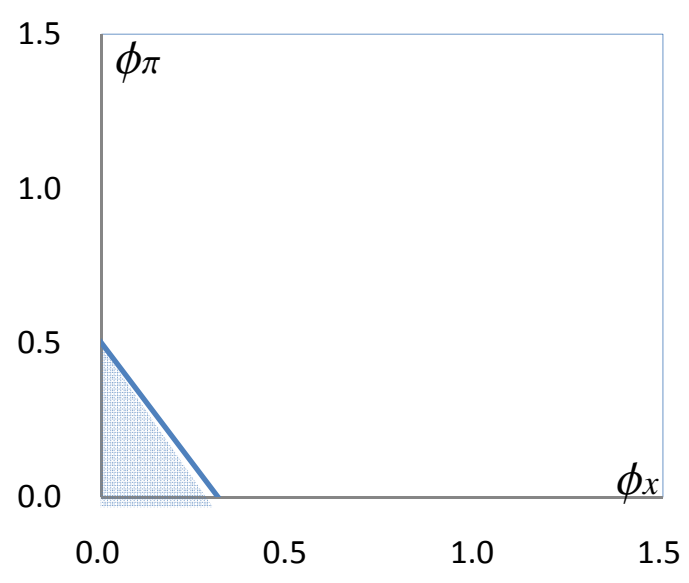

(b) Heterogeneous Learning

$$
(n=2, \rho g u=1.0)
$$

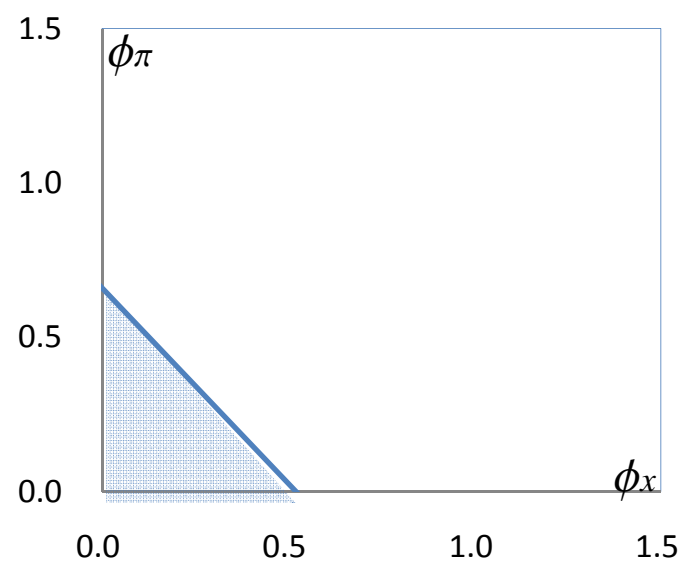

(d) Heterogeneous Learning

$$
(n=2, \rho g u=0.7)
$$

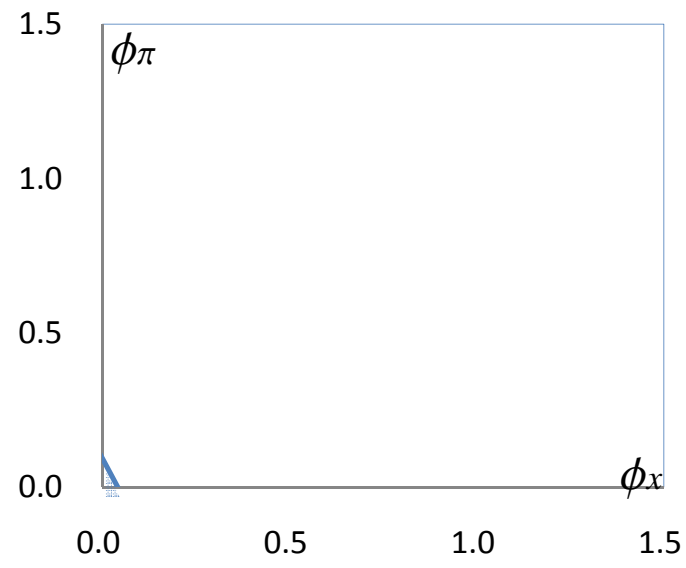

Note: This figure shows the values of policy parameters $\left(\phi \pi, \phi_{x}\right)$ to ensure the stability of the equilibrium under adaptive learning and the effect of correlation of demand and supply shocks $(\rho g u)$. In each panel, the bright area represents the region of the parameters satisfying the stability conditions. 


\section{Figure 2 Dynamics of estimates $\left(\mathrm{a}_{\mathrm{t}}, \mathrm{c}_{\mathrm{t}}\right)$ under $\phi_{\pi}=5$ and $\rho_{\mathrm{gu}}=0.75$}
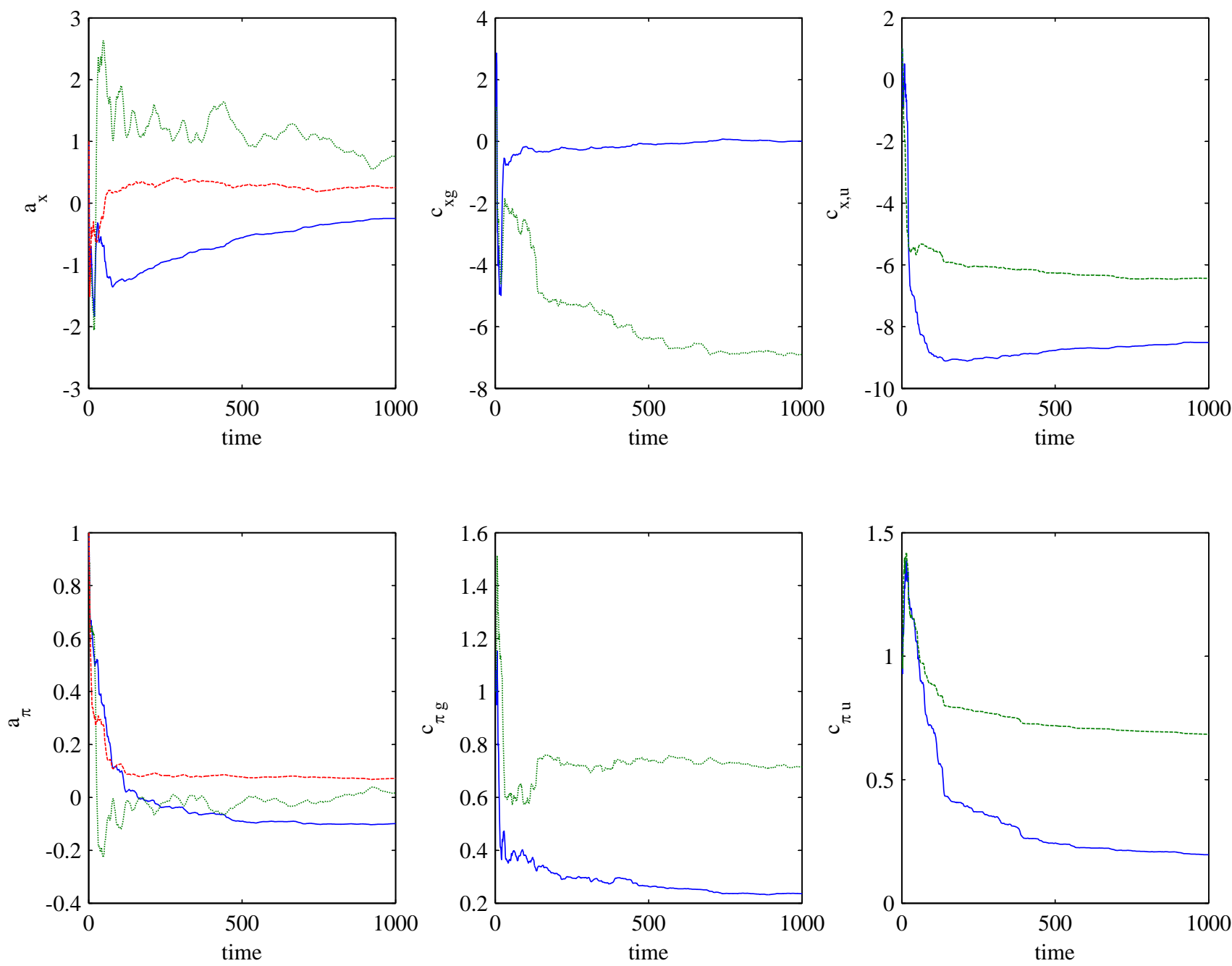

Note: This figure shows the dynamics of the estimates when the central bank chooses an active policy (phi_pi $=5$ ), which satisfies stability conditions under both homogeneous and heterogeneous learning rules with constant terms. Solid lines (blue) represent the transition of estimates under homogeneous learning. Dotted (black) and dashed (red) lines represent the transition of estimates of agents of Type 1 and 2 under heterogeneous learning, respectively. Under heterogeneous learning, (a_x, a_pi) is estimated by both types of agents, whereas ( $c \_x g, c \_$pi $g$ ) and (c_xu, c_pi $u$ ) are respectively estimated by agents of Type 1 and 2 . The dynamics of $c$ under learning rules without constant terms is almost the same as the above dynamics of $c$. 


\section{Figure 3 Dynamics of estimates $\left(\mathrm{a}_{\mathrm{t}}, \mathrm{c}_{\mathrm{t}}\right)$ under $\phi_{\pi}=0.5$ and $\rho_{\mathrm{gu}}=0.75$}
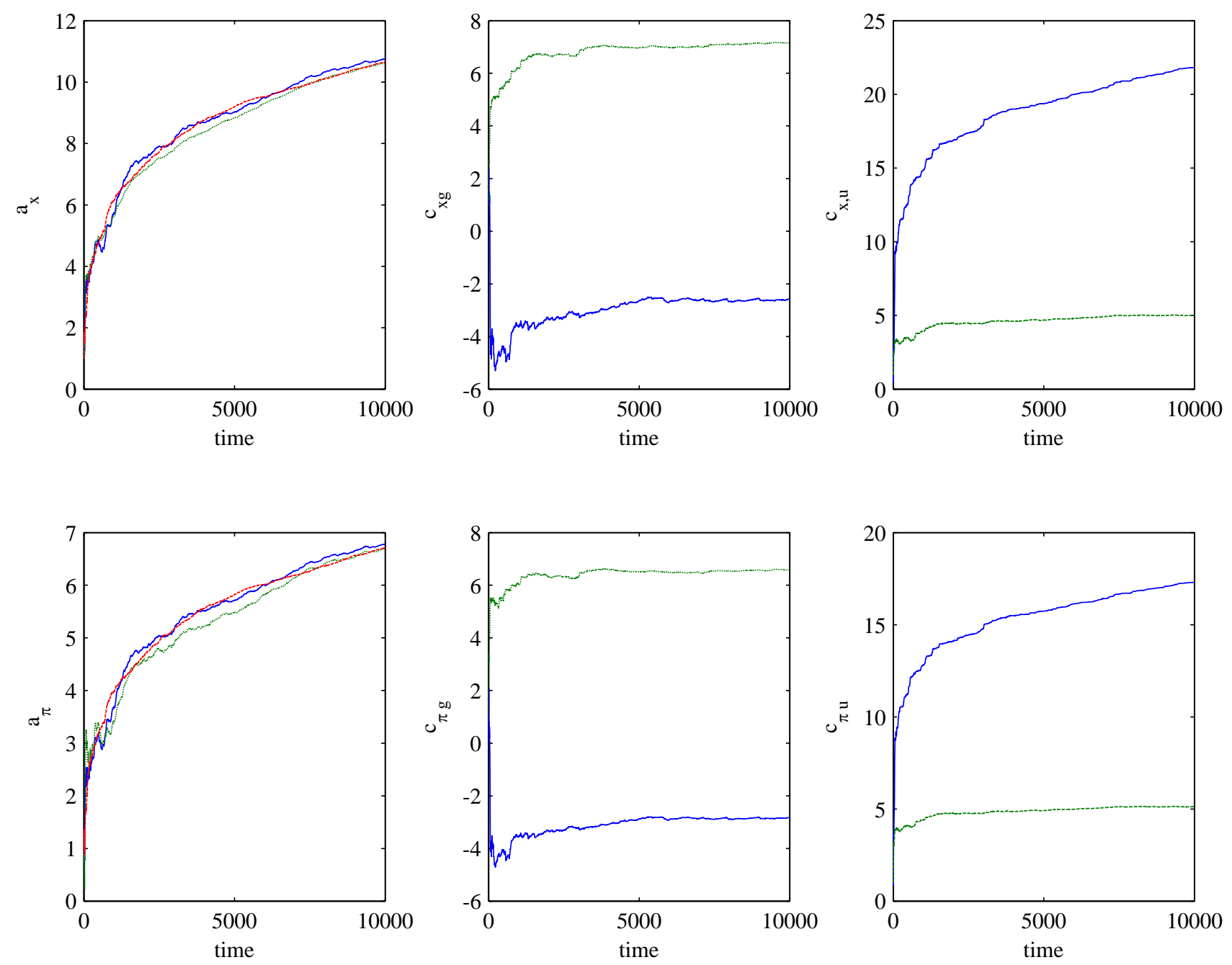

Note: This figure shows the dynamics of the estimates when the central bank chooses a passive policy (phi_pi $=0.5$ ), which does not satisfy the stability condition under a homogeneous learning rule without constant terms, but satisfies the condition under heterogeneous learning rules without constant terms. Other details are seen in Figure 2. 
Figure 4 Dynamics of estimates $\left(\mathrm{a}_{\mathrm{t}}, \mathrm{c}_{\mathrm{t}}\right)$ under $\phi_{\pi}=0.1$ and $\rho_{\mathrm{gu}}=0.75$
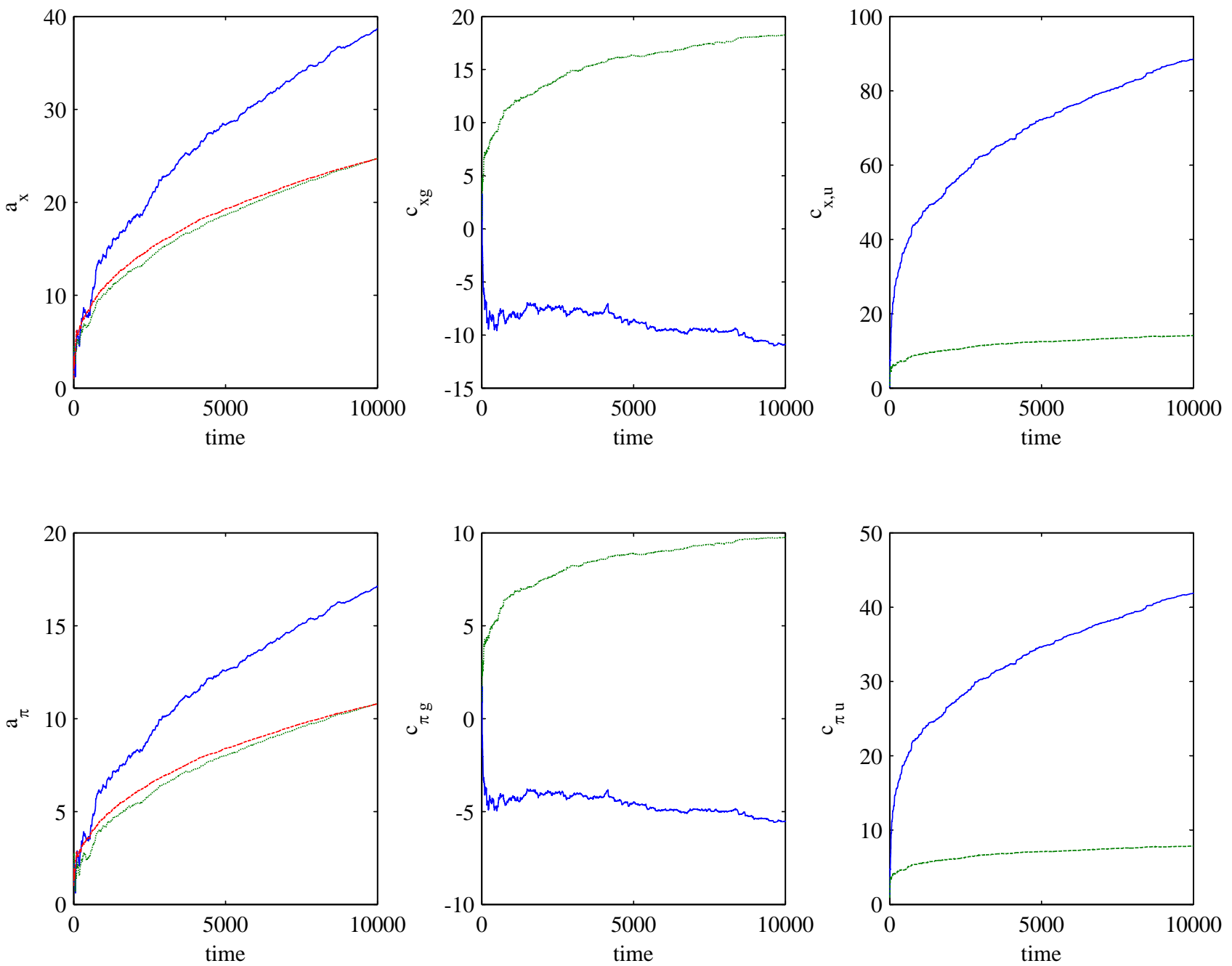

Note: This figure shows the dynamics of the estimates when the central bank chooses a further passive policy (phi_pi $=0.1$ ), which satisfies the stability conditions under neither homogeneous learning nor under heterogeneous learning. Other details are seen in Figure 2. 


\section{Figure 5 Dynamics of estimates $\left(\mathrm{a}_{\mathrm{t}}, \mathrm{c}_{\mathrm{t}}\right)$ under $\phi_{\pi}=0.1$ and $\rho_{\mathrm{gu}}=0$}
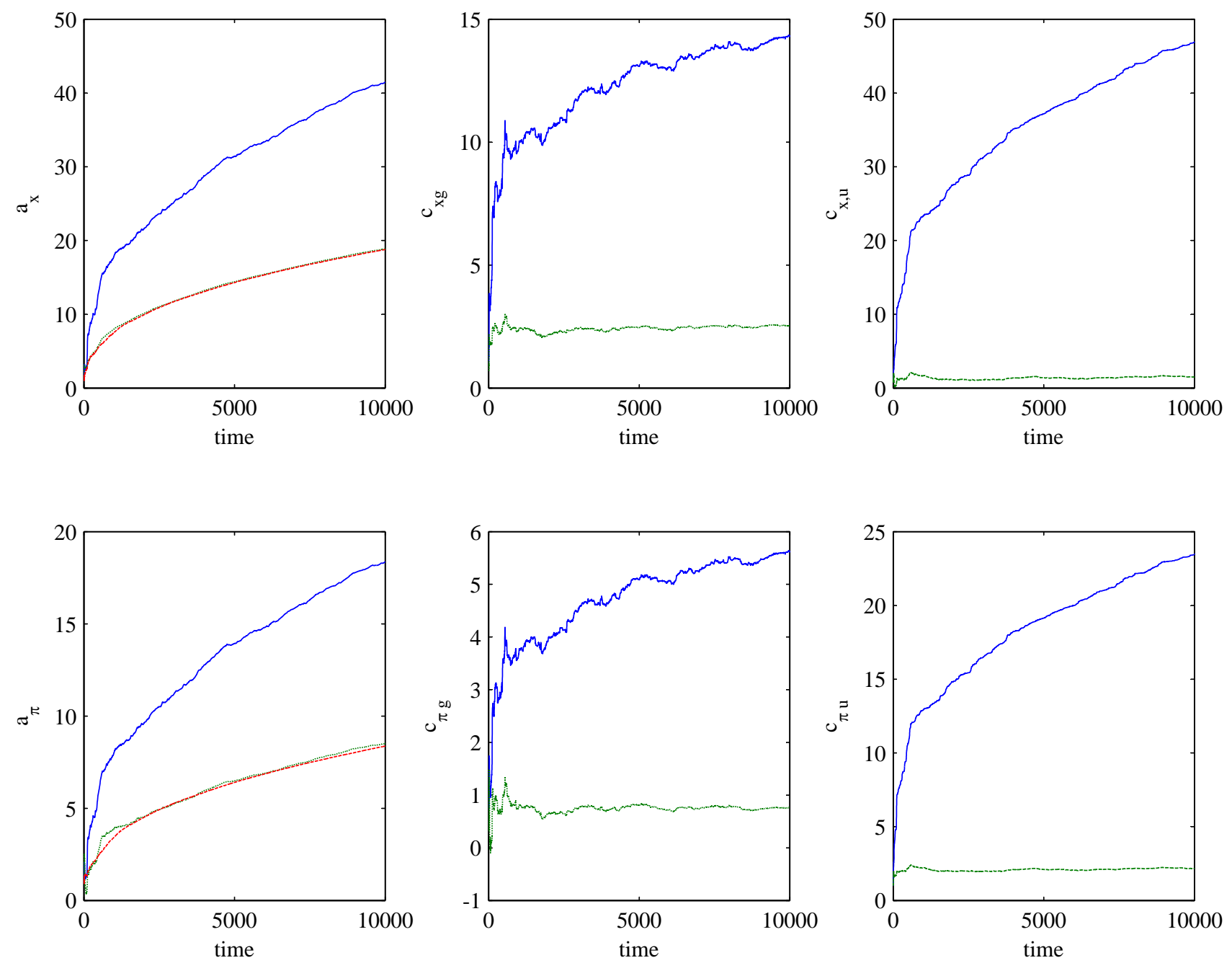

Note: This figure shows the dynamics of the estimates where the correlation of demand and supply shocks is low $($ rho $=0)$. The central bank chooses a further passive policy (phi pi $=0.1$ ), which does not satisfy the stability condition under a homogeneous learning rule without constant terms, but satisfies the condition under heterogeneous learning rules without constant terms. Other details are seen in Figure 2. 